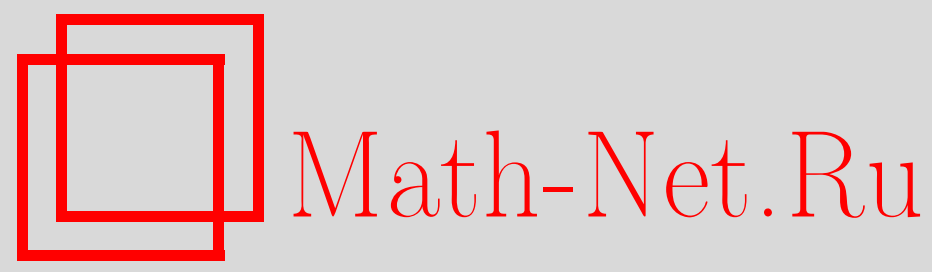

Н. С. Кучеренко, Средняя сложность поиска идентичных объектов для случайных неравномерных баз данных, Дискрет. матем., 2011, том 23, выпуск 2, 129-158

DOI: https://doi.org/10.4213/dm1148

Использование Общероссийского математического портала Math-Net.Ru подразумевает, что вы прочитали и согласны с пользовательским соглашением http://www . mathnet.ru/rus/agreement

Параметры загрузки:

IP: 34.229 .45 .116

26 апреля 2023 г., $14: 37: 14$ 
УДК 519.2

\title{
Средняя сложность поиска идентичных объектов для случайных неравномерных баз данных
}

\author{
() 2011 г. Н.С.Кучеренко
}

\begin{abstract}
В работе рассматривается поведение средней сложности оптимальных алгоритмов решения задачи поиска идентичных объектов (ЗПИО) для случайных баз данных. Описаны и исследованы классы ЗПИО, для которых функция роста средней сложности как функция от объема базы данных имеет логарифмический порядок роста. Для таких классов задач получены точные асимптотики функций роста. Изучен случай, когда сложность оптимального алгоритма в среднем по классу задач ограничена. Построен класс ЗПИО, для которого функция роста средней сложности оптимального алгоритма является неограниченной функцией по порядку меньшей логарифма.
\end{abstract}

\section{1. Введение}

Теория хранения и поиска информации является важным разделом теории интеллектуальных систем. Одним из ключевых объектов этой теории является информационный граф (ИГ) - управляющая система, которая позволяет рассматривать имеющиеся модели данных и задачи, связанные с ними, с более общих позиций [1].

В работе рассматривается задача поиска идентичных объектов (ЗПИО), которая является одним из способов формализации задачи поиска по ключу [6]. Практически такая задача возникает, когда на объектах базы данных введен линейный порядок. Предполагается, что объекты рассортированы в соответствии с этим порядком и по пришедшему запросу необходимо найти соответствующий ему объект массива. Алгоритмы поиска, использующие только операции сравнения, исследуются с точки зрения сложности, которая характеризует среднее время работы алгоритма.

Идея представлять такие алгоритмы поиска с помощью деревьев возникла в пятидесятые годы двадцатого века. В 1959 году Э. Н. Гильберт и Э. Ф. Мур показали, что можно построить оптимальное дерево поиска за порядка $n^{3}$ шагов ( $n-$ мощность базы данных), и привели оценки сложности такого дерева поиска [7]. В 1971 году Д. Э. Кнут показал, что построение оптимального дерева поиска можно улучшить до порядка $n^{2}$ шагов [6]. Дальнейшие упрощения методов построения были произведены в 1977 году А. М. Гарсия и М. Л. Вочем. Их метод позволяет построить оптимальное дерево поиска за $O\left(n \log _{2} n\right)$ шагов [8].

В данной работе алгоритмы поиска представляются с помощью информационных графов. Для любого объема базы данных $n$ сложность оптимального ИГ не больше $\log _{2}(n+1)$ и не меньше единицы [1]. При этом существуют такие ЗПИО, на которых эти оценки 
достигаются. Поэтому возникает вопрос о поведении средней сложности оптимального информационного графа на классах задач. В [2] рассмотрен класс задач, для которых база данных - случайный вектор, компоненты которого равномерно распределены на интервале $(0,1)$, запросы также распределены равномерно на интервале $(0,1)$. Показано, что для любого объема базы данных $n$ в среднем по классу задач сложность оптимального ИГ незначительно отличается от $\log _{2}(n+1)$.

В данной работе рассматриваются классы задач, у которых распределение элементов данных и запросов может быть отлично от равномерного распределения. Распределение элементов данных и запросов задаются с помощью функций плотности $g$ и $f$ соответственно.

Получено достаточное условие на функции $f$ и $g$, при котором в среднем по классу задач сложность оптимального ИГ имеет порядок логарифма. Этим условием является существование интервала ненулевой длины, на котором функции плотности $f$ и $g$ одновременно ограничены и отделены от нуля. Асимптотическое поведение функции роста средней сложности оптимального ИГ можно описать, когда функции плотности интегрируемы по Риману и либо ограничены и отделены от нуля на всем интервале $(0,1)$, либо могут определенным образом принимать нулевое значение. В первом случае функция роста асимптотически ведет себя как двоичный логарифм от объема базы данных, во втором случае перед логарифмом возникает константа, которая зависит только от функций $f$ и $g$.

Также в работе рассмотрены классы задач, функции плотности $f$ и $g$ которых не удовлетворяют описанному выше достаточному условию. Показано, что для любого отрезка $[b, b+2], b \in \mathbf{R}, b>1$, существуют такие функции плотности, что при достаточно большом объеме базы данных функция роста средней сложности оптимального ИГ не выходит за пределы этого отрезка. Показано, что существуют такие функции плотности, для которых средняя сложность оптимального ИГ равна единице для любого объема базы данных.

Возможное поведение функции роста средней сложности оптимального ИГ может отличаться от описанного выше логарифмического и ограниченного случая. Построены такие функции плотности, что функция роста по порядку не больше $\ln \ln n$, но не меньше $\ln \ln \ln n$ при увеличении объема базы данных $n$.

Автор выражает благодарность своему научному руководителю профессору Э. Э. Гасанову за постановку задачи и внимание к работе и академику В. Б. Кудрявцеву за ценные советы и замечания.

\section{2. Основные понятия и результаты}

В данной работе исследуются алгоритмы поиска, использующие только операции сравнения. Ввиду этого, будет использоваться упрощенная версия терминологии из книги [1].

Задача поиска идентичных объектов - это четверка $((0,1), V, \rho=, f(x))$, где $(0,1)$ - множество запросов, $V=\left(y_{1}, y_{2}, \ldots, y_{n}\right)$ - конечный набор точек интервала $(0,1)$, элементы которого упорядочены по возрастанию. Множество $V$ называется библиотекой, а элементы библиотеки - записями. Отношение $\rho=-$ отношение равенства на множестве $(0,1) \times(0,1)$. Предполагается, что запрос $X \in(0,1)-$ случайная величина, которая принимает значения из интервала $(0,1)$, и распределение которой задается с помощью функции плотности $f(t)$. Для реализации $x$ случайной величины $X$ отношение $\rho=$ задает 
функцию ответов $J_{I}(x)$ следующим образом:

$$
J(x)= \begin{cases}\left\{y_{i}\right\}, & \text { если найдется } i \in[1, \ldots, n], \text { такое, что } y_{i} \rho_{=} x, \\ \varnothing & \text { в противном случае. }\end{cases}
$$

Для реализации функции ответов используются операции сравнения вещественных чисел из интервала $(0,1)$, которые заданы в виде функции

$$
p(y, x)=J_{I}(x)= \begin{cases}1, & \text { если } x<y, \\ 2, & \text { если } x=y, \\ 3, & \text { если } x>y,\end{cases}
$$

для всех $y, x \in(0,1)$.

Функция $p_{a}(x)=p(a, x), a \in(0,1)$, называется переключателем. Определим базовое множество переключателей

$$
G=\left\{p_{a}(x) \mid a \in(0,1)\right\} .
$$

Над базовым множеством $G$ строится информационный граф (ИГ), который хранит в себе схему вызова переключателей из $G$.

Информационный граф - это связный ориентированный конечный граф без кратных ребер и петель, вершины и ребра которого размечены следующим образом. Выделены два подмножества вершин $P$ и $L$ так, что $P \cap L=\varnothing$. Вершины множества $P$ называются переключательными, а вершины множества $L$ - листьями. Ребра, исходящие из переключательных вершин, называются переключательными. Среди переключательных вершин выделена одна вершина $v_{0}$, которая называется корневой. Переключательным вершинам сопоставлены переключатели из $G$. Переключатель $p_{a}(x)$, сопоставленный переключательной вершине $v$, называется ее нагрузкой и обозначается $[v]_{P}=p_{a}(x)$. Листьям сопоставляются записи библиотеки. Это сопоставление обозначается $[v]_{L}=y_{i}, v \in L$; $y_{i}$ называется нагрузкой листа $v$. Переключательным ребрам сопоставляются элементы множества $\{\Lambda, 1,2,3\}$, при этом из переключательной вершины не выходит двух ребер с одинаковыми номерами. Если ребру $\left(v_{i_{1}}, v_{i_{2}}\right)$ сопоставлен номер $i \in\{1,2,3\}$, то номер $i$ называется нагрузкой ребра $\left(v_{i_{1}}, v_{i_{2}}\right)$ и обозначается $\left[\left(v_{i_{1}}, v_{i_{2}}\right)\right]=i$.

Определим функционирование информационного графа. Переключательное ребро $\left(v_{i_{1}}, v_{i_{2}}\right)$ проводит запрос $x$, если выполнено условие

$$
\left[\left(v_{i_{1}}, v_{i_{2}}\right)\right]=\left[v_{i_{1}}\right]_{P}(x) .
$$

Непереключательное ребро не проводит запрос, также не проводит запрос переключательное ребро, которому был сопоставлен символ $\Lambda$. Ориентированная цепочка ребер проводит запрос $x$, если каждое ребро цепочки проводит запрос $x$. Запрос $x$ проходит в вершину $v$ ИГ, если существует ориентированная цепочка, ведущая из корневой вершины в вершину $v$, которая проводит запрос $x$. Запись $y$, приписанная листу $v^{\prime}$, называется результатом функционирования ИГ на запросе $x$, если запрос $x$ проходит в лист $v^{\prime}$. Результат функционирования ИГ $U-$ это функция $R_{U}(x)$, которая каждому запросу $x$ сопоставляет множество всех записей, которые являются результатом функционирования ИГ $U$ на запросе $x$.

Информационный граф $U$ решает ЗПИО $I$, если результат функционирования ИГ $U$ совпадает с функцией ответов $J_{I}(x)$, то есть для любых $x \in(0,1)$

$$
R_{U}(x)=J_{I}(x) .
$$


Если в информационном графе $U$ удалить нефункциональные элементы, а именно, непереключательные ребра и ребра, которым сопоставлен символ $\Lambda$, а затем оставить только одну компоненту связности, которой принадлежит корневая вершина, то результат функционирования $R_{U}(x)$ не изменится. Поэтому далее рассматриваются ИГ с точностью до этих операций удаления.

Множество ИГ, которые решают ЗПИО $I$, обозначим через $S_{I}$. Сложностью ИГ $U$ на запросе $x$ называется число

$$
T(U, x)=\sum_{v \in P} \varphi_{v}(x)
$$

где $P$ - множество переключательных вершин, а $\varphi_{v}(x)$ - предикат, принимающий значение единица, если $x$ проходит в вершину $v$, и нуль в противном случае. Предикат $\varphi_{v}(x)$ называется функцией фильтра вершины $v$. Величина $T(U, X)$ как функция от случайной величины $X$ также является случайной величиной [1]. Математическое ожидание величины $T(U, X)$ называется сложностью ИГ $U$ для ЗПИО $I$ и обозначается $T_{I}(U)$.

Сложностью ЗПИО I называется величина

$$
T(I)=\inf _{U \in S_{I}} T_{I}(U) .
$$

Информационный граф, на котором достигается инфимум, называется оптимальным информационным графом.

В работе рассмотрен класс задач поиска идентичных объектов, который задается с помощью случайной библиотеки

$$
\tilde{V}=\left(Y_{(1)}, Y_{(2)}, \ldots, Y_{(n)}\right),
$$

где $Y_{(1)}, Y_{(2)}, \ldots, Y_{(n)}$ - вариационный ряд независимых случайных величин $Y_{1}, Y_{2}, \ldots, Y_{n}$ с функцией плотности распределения $g(t)$.

Обозначим через $T_{n}^{(f, g)}(V)$ сложность ЗПИО $((0,1), V, \rho=, f)$, где библиотека $V-$ реализация случайной библиотеки $\widetilde{V}$, элементы которой имеют функцию плотности $g(t)$. Рассмотрим случайную величину $T_{n}^{(f, g)}(\widetilde{V})$, ее математическое ожидание обозначим через $\mathbf{M}_{\widetilde{V}}\left(T_{n}^{(f, g)}(\tilde{V})\right)$.

Если известно, что функции плотности $f$ и $g$ на некоторых интервалах ограничены и отделены от нуля, то можно привести следующие оценки.

Теорема 1. Пусть функции плотности удовлетворяют следующим ограничениям: существует $s, s \in \mathbf{N}$, непересекающихся интервалов $\left(a_{i}, b_{i}\right) \subset(0,1), i=1, \ldots, s$, таких что для всех $i=1, \ldots$, s найдутся $e_{1}^{i}, e_{2}^{i}, k_{1}^{i}, k_{2}^{i} \in \mathbf{R}^{+}$, такие, что

$$
e_{1}^{i} \geqslant f(x) \geqslant e_{2}^{i}>0, \quad k_{1}^{i} \geqslant g(x) \geqslant k_{2}^{i}>0
$$

для произвольных $x \in\left(a_{i}, b_{i}\right)$. Тогда для любого вещественного положительного $\varepsilon$ существует такое натуральное $n_{0}$, что для любого $n$, превосходящего $n_{0}$, справедливо неравенство

$$
\mathbf{M}_{\tilde{V}}\left(T_{n}^{(f, g)}(\tilde{V})\right) \geqslant \sum_{i=1}^{s} \frac{e_{2}^{i} r_{i}}{k_{1}^{i}} \log _{2}\left(r_{i} n+1\right)-\frac{1-\gamma}{\ln 2} \sum_{i=1}^{s} \frac{e_{2}^{i} r_{i}}{k_{1}^{i}}-\varepsilon,
$$

гेe

$$
r_{i}=\int_{a_{i}}^{b_{i}} g(x) d x
$$

и $\gamma$ - постоянная Эйлера. 
Следствием из теоремы 1 является достаточное условие на функции плотности $f$ и $g$, при котором математическое ожидание сложности поиска имеет порядок логарифма от мощности библиотеки.

Следствие 1. Если существует интервал, на котором функции плотности $f$ u $g$ одновременно ограничены и отделены от нуля, то при $n \rightarrow \infty$

$$
\mathbf{M}_{\tilde{V}}\left(T_{n}^{(f, g)}(\tilde{V})\right) \asymp \log _{2} n .
$$

Получены условия на функции плотности $f$ и $g$, при которых известна асимптотика математического ожидания сложности поиска $\mathbf{M}_{\widetilde{V}}\left(T_{n}^{(f, g)}(\tilde{V})\right)$. Обозначим через $\operatorname{supp}(f)$ множество точек интервала $(0,1)$, на которых функция $f$ отлична от нуля. Назовем функцию $f$ правильной, если $\operatorname{supp}(f)$ имеет вид

$$
\operatorname{supp}(f)=\bigsqcup_{i=1}^{s}\left(a_{i}, b_{i}\right) \sqcup K, \quad K \subseteq\left\{a_{1}, \ldots, a_{s}, b_{1}, \ldots, b_{s}\right\},
$$

где $a_{i}<b_{i}, b_{i}<a_{i+1}, i=1, \ldots, s-1, a_{s}<b_{s}$.

Теорема 2. Пусть функичии плотности $f$ и я являются правильными функциями, которые ограничены и отделены от нуля на множестве $\operatorname{supp}(f) u \operatorname{supp}(g)$ соответственно, и существует интервал, на котором функщии ограничены и отделены от нуля одновременно. Пусть также функиии $f$ и $g$ интегрируемы по Риману. Тогда при $n \rightarrow \infty$

$$
\mathbf{M}_{\tilde{V}}\left(T_{n}^{(f, g)}(\tilde{V})\right) \sim \log _{2} n \int_{B} f(x) d x,
$$

где $B=\operatorname{supp}(g)$.

Следствие 2. Пусть функции плотности $f$ u $g$ интегрируемы по Риману, ограничены $и$ отделены от нуля на всем интервале $(0,1)$. Тогда при $n \rightarrow \infty$

$$
\mathbf{M}_{\tilde{V}}\left(T_{n}^{(f, g)}(\tilde{V})\right) \sim \log _{2} n .
$$

Функция роста математического ожидания сложности поиска может быть по порядку отлична от логарифма. В следующей теореме изучаются ограниченные функции роста величины $\mathbf{M}_{\widetilde{V}}\left(T_{n}^{(f, g)}(\tilde{V})\right)$.

Теорема 3. Сущесствуют такие функции плотности $f^{\prime}$ и $g^{\prime}$, что для всех $n \in \mathbf{N}$

$$
\mathbf{M}_{\tilde{V}}\left(T_{n}^{\left(f^{\prime}, g^{\prime}\right)}(\tilde{V})\right)=1 .
$$

Для любого вещчественного $b>1$ сущеествуют такие функичи плотности $f^{\prime \prime} u g^{\prime \prime}, u$ сушествует такое натуральное $n_{0}$, что для всех $n>n_{0}$

$$
b+2 \leqslant \mathbf{M}_{\widetilde{V}}\left(T_{n}^{\left(f^{\prime \prime}, g^{\prime \prime}\right)}(\tilde{V})\right) \leqslant b .
$$

Функции роста математического ожидания сложности поиска могут быть неограниченно возрастающими, но по порядку меньшими логарифма. В следующей теореме строятся функции плотности $f$ и $g$, для которых такое поведение имеет место.

Теорема 4. Существуют такие функции плотности $f$ и $g$, что при $n \rightarrow \infty$

$$
\frac{\ln 2}{a} \ln \ln n \gtrsim \mathbf{M}_{\widetilde{V}}\left(T_{n}^{(f, g)}(\widetilde{V})\right) \gtrsim \frac{\ln 2}{a} \ln \ln \ln n,
$$

2de

$$
a=\sum_{i=2}^{\infty} \frac{1}{i \log _{2}^{2} i} .
$$




\section{3. Доказательство теоремы 1}

Реализацию вариационного ряда $Y_{(1)}, \ldots, Y_{(n)}$ обозначим через $y_{(1)}, \ldots, y_{(n)}$. Рассмотрим ЗПИО $\left((0,1),\left(y_{(1)}, \ldots, y_{(n)}\right), \rho=, f\right)$. Точки 0 и 1 обозначим через $y_{(0)}$ и $y_{(n+1)}$ соответственно.

Обозначим через $p_{i}$ вероятность того, что запрос попадет в интервал $\left(y_{(i)}, y_{(i+1)}\right)$. Поскольку функция распределения запросов имеет плотность, для всех $i=0,1, \ldots, n$

$$
p_{i}=\int_{y_{(i)}}^{y_{(i+1)}} f(t) d t
$$

Обозначим через $F$ и $G$ функции распределения, которые определяются функциями плотности $f$ и $g$ соответственно.

Если функции плотности распределения запроса $f$ и записи библиотеки $g$ для всех $t \in(0,1)$ удовлетворяют условиям

$$
h_{1} \geqslant f(t) \geqslant h_{2}>0, \quad h_{1}^{\prime} \geqslant g(t) \geqslant h_{2}^{\prime}>0,
$$

то записи библиотеки как случайные величины можно моделировать методом обратной функции. Обозначим через $Z_{1}, \ldots, Z_{n}$ независимые равномерно распределенные случайные величины. Через $Z_{(1)}, \ldots, Z_{(n)}$ обозначим их вариационный ряд. Случайные величины $Y_{1}=G^{-1}\left(Z_{1}\right), \ldots, Y_{n}=G^{-1}\left(Z_{n}\right)$ независимы и имеют функцию распределения $G(x)$. Поскольку функция $G^{-1}(x)$ монотонна, справедливы равенства

$$
Y_{(1)}=G^{-1}\left(Z_{(1)}\right), \ldots, Y_{(n)}=G^{-1}\left(Z_{(n)}\right) .
$$

Вероятность $p_{i}$ оценивается через разность реализаций $\left(z_{(i+1)}-z_{(i)}\right)$ случайных величин $Z_{(i)}, Z_{(i+1)}$ следующим образом. Положим $z_{(0)}=0, z_{(n+1)}=1$.

Лемма 1. Если функциии плотности таковы, что для произвольного $t \in(0,1)$

$$
h_{1} \geqslant f(t) \geqslant h_{2}>0, \quad h_{1}^{\prime} \geqslant g(t) \geqslant h_{2}^{\prime}>0,
$$

то для всех $i=0, \ldots, n$

$$
\frac{h_{1}}{h_{2}^{\prime}}\left(z_{(i+1)}-z_{(i)}\right) \geqslant p_{i} \geqslant \frac{h_{2}}{h_{1}^{\prime}}\left(z_{(i+1)}-z_{(i)}\right) .
$$

Доказательство. Поскольку $h_{1} \geqslant f(x) \geqslant h_{2}>0$, из представления вероятности $p_{i}$ в виде интеграла $\int_{y_{(i)}}^{y_{(i+1)}} f(x) d x$ следует, что для всех $i=0, \ldots, n$

$$
h_{1}\left(y_{(i+1)}-y_{(i)}\right) \geqslant p_{i} \geqslant h_{2}\left(y_{(i+1)}-y_{(i)}\right) .
$$

Разность $\left(y_{(i+1)}-y_{(i)}\right)$ можно записать в виде

$$
y_{(i+1)}-y_{(i)}=G^{-1}\left(z_{(i+1)}\right)-G^{-1}\left(z_{(i)}\right) .
$$

Поскольку производная функции $G^{-1}(t)$ равна $1 / g(t)$ и функция $g(t)$ ограничена по условию леммы, верны оценки

$$
\frac{1}{h_{2}^{\prime}}\left(z_{(i+1)}-z_{(i)}\right) \geqslant y_{(i+1)}-y_{(i)} \geqslant \frac{1}{h_{1}^{\prime}}\left(z_{(i+1)}-z_{(i)}\right) .
$$


Приходим к цепочке неравенств, верных при $i=0,1, \ldots, n$ :

$$
\frac{h_{1}}{h_{2}^{\prime}}\left(z_{(i+1)}-z_{(i)}\right) \geqslant h_{1}\left(y_{(i+1)}-y_{(i)}\right) \geqslant p_{i} \geqslant h_{2}\left(y_{(i+1)}-y_{(i)}\right) \geqslant \frac{h_{2}}{h_{1}^{\prime}}\left(z_{(i+1)}-z_{(i)}\right) .
$$

Лемма доказана.

Рассмотрим функцию энтропии

$$
H\left(p_{0}, \ldots, p_{n}\right)=\sum_{i=0}^{n}\left(-p_{i} \log _{2} p_{i}\right)
$$

Изучим, при каком условии, подставляя нижние оценки для вероятностей $p_{i}$, мы можем получить нижнюю оценку для всей функции энтропии. Функция энтропии - это сумма слагаемых вида $-\left(x \log _{2} x\right)$. Функция $-\left(x \log _{2} x\right)$ возрастает на интервале $(0,1 / e)$ и убывает на интервале $(1 / e, 1)$. Если обозначить через $p_{(n)}$ максимальное значение среди всех $p_{i}$, то искомое условие записывается в виде $p_{(n)}<1 / e$.

Покажем, что если функции плотности ограничены и отделены от нуля на всем интервале $(0,1)$, то вероятность события $\left\{p_{(n)}<1 / e\right\}$ стремится к единице с ростом числа записей библиотеки $n$. Обозначим расстояние между точками $z_{(i)}$ и $z_{(i+1)}$ через $\delta_{i}$. Введем обозначение

$$
\delta_{(n)}=\max _{0 \leqslant i \leqslant n}\left(\delta_{i}\right) .
$$

Лемма 2. Пусть функции плотности $f$ u g удовлетворяют следующчим условиям: для произвольного $x \in(0,1)$

$$
h_{1} \geqslant f(x) \geqslant h_{2}>0, \quad h_{1}^{\prime} \geqslant g(x) \geqslant h_{2}^{\prime}>0 .
$$

Тогда для любого натурального $n, n>2[1 / a]-1$, справедливо неравенство

$$
\mathbf{P}\left(p_{(n)} \leqslant 1 / e\right) \geqslant \mathbf{P}\left(\delta_{(n)} \leqslant \frac{1}{e} \frac{h_{2}^{\prime}}{h_{1}}\right) \geqslant 1-(1-a)^{n}(n+1)^{b},
$$

где

$$
a=\frac{h_{2}^{\prime}}{h_{1} e}<1, \quad b=\left[\frac{1}{a}\right]+1>1 .
$$

Доказательство. В силу леммы 1 ,

$$
\frac{h_{1}}{h_{2}^{\prime}} \delta_{i} \geqslant p_{i}
$$

для всех $i=0,1, \ldots, n$. Из неравенства

$$
\frac{h_{1}}{h_{2}^{\prime}} \delta_{(n)} \leqslant \frac{1}{e}
$$

следует, что $p_{(n)} \leqslant 1 / e$. Поэтому

$$
\mathbf{P}\left(p_{(n)} \leqslant \frac{1}{e}\right) \geqslant \mathbf{P}\left(\frac{h_{1}}{h_{2}^{\prime}} \delta_{(n)} \leqslant \frac{1}{e}\right) .
$$


Вероятность $\mathbf{P}\left(\delta_{(n)} \leqslant a\right)$ можно записать следующим образом [4]:

$$
\mathbf{P}\left(\delta_{(n)} \leqslant a\right)=\sum_{i=0}^{n+1}(-1)^{i}\left(\begin{array}{c}
n+1 \\
i
\end{array}\right)(1-i a)^{n} I_{\{(1-i a)>0\}},
$$

где функция $I_{\{(1-i a)>0\}}$ равна единице, если равенство в фигурных скобках верно, и нулю в противном случае. Исследуем правую часть равенства при $a=h_{2}^{\prime} /\left(h_{1} e\right)$. Заметим, что $a<1$.

Поскольку условие $(1-i a)>0$ верно при $i<1 / a$, то правую часть равенства можно записать в виде

$$
\sum_{i=0}^{n+1}(-1)^{i}\left(\begin{array}{c}
n+1 \\
i
\end{array}\right)(1-i a)^{n} I_{\{(1-i a)>0\}}=1-\sum_{i=1}^{[1 / a]}(-1)^{i+1}\left(\begin{array}{c}
n+1 \\
i
\end{array}\right)(1-i a)^{n} .
$$

Оценим абсолютное значение выражения $\sum_{i=1}^{[1 / a]}(-1)^{i+1}\left(\begin{array}{c}n+1 \\ i\end{array}\right)(1-i a)^{n}$. Заметим, что при выполнении условия $1 \leqslant i \leqslant[1 / a]$ верно неравенство $(1-i a)^{n} \leqslant(1-a)^{n}$. Для оценивания биномиальных коэффициентов возьмем такое натуральное $n_{0}$, что для любого $n>n_{0}$ выполнено неравенство $(n+1) / 2>[1 / a]$. Положим $n_{0}=2[1 / a]-1$, тогда при $n>2[1 / a]-1$ и при всех $i=1,2, \ldots,([1 / a]-1)$ выполнено неравенство

$$
\left(\begin{array}{c}
n+1 \\
i
\end{array}\right)<\left(\begin{array}{c}
n+1 \\
{[1 / a]}
\end{array}\right)
$$

Оценим биномиальный коэффициент $\left(\begin{array}{c}n+1 \\ {[1 / a]}\end{array}\right)$ следующим образом:

$$
\left(\begin{array}{c}
n+1 \\
{[1 / a]}
\end{array}\right)=\frac{(n+1) \cdots(n+1-[1 / a])}{[1 / a] !}<\frac{1}{[1 / a]}(n+1)^{[1 / a]+1} .
$$

Подставляя полученные оценки в исследуемое выражение, получаем, что при $n>2[1 / a]-1$

$$
\left|\sum_{i=1}^{[1 / a]}(-1)^{i+1}\left(\begin{array}{c}
n+1 \\
i
\end{array}\right)(1-i a)^{n}\right|<(n+1)^{[1 / a]+1}(1-a)^{n} .
$$

Следовательно, при $n>2[1 / a]-1$ справедливы неравенства

$$
\mathbf{P}\left(\delta_{(n)} \leqslant a\right) \geqslant 1-(1-a)^{n}(n+1)^{[1 / a]+1},
$$

где $a=h_{2}^{\prime} /\left(h_{1} e\right)$. Лемма доказана.

Для любой реализации $V=\left(y_{(1)}, \ldots, y_{(n)}\right)$ библиотеки $\tilde{V}=\left(Y_{(1)}, \ldots, Y_{(n)}\right)$ верно неравенство [2]

$$
T\left((0,1),\left(y_{(1)}, \ldots, y_{(n)}\right), \rho_{=}, f\right) \geqslant H\left(p_{0}, \ldots, p_{n}\right) .
$$

Положим $Y_{(0)}=0$ и $Y_{(n+1)}=1$ и введем случайные величины

$$
P_{i}=\int_{y_{(i)}}^{y_{(i+1)}} f(x) d x, \quad i=0,1, \ldots, n .
$$


Обозначим через $H_{n}^{(f, g)}(\tilde{V})$ величину $H\left(P_{0}, \ldots, P_{n}\right)$ и назовем ее нижней энтропийной оценкой. Верно следующее неравенство

$$
\mathbf{M}_{\widetilde{V}}\left(T_{n}^{(f, g)}(\tilde{V})\right) \geqslant \mathbf{M}_{\widetilde{V}}\left(H_{n}^{(f, g)}(\tilde{V})\right) .
$$

Оценим математическое ожидание нижней энтропийной оценки при условии, что функции плотности $f$ и $g$ ограничены и отделены от нуля на интервале $(0,1)$. Введем следующие обозначения:

$$
Y_{(i+1)}-Y_{(i)}=\Delta_{i}, \quad i=0,1, \ldots, n, \quad \Delta_{(n)}=\max _{0 \leqslant i \leqslant n} \Delta_{i}
$$

Лемма 3. Пусть для произвольного $t \in(0,1)$ функиии плотности $f$ и $g$ удовлетворяют ограничениям

$$
h_{1} \geqslant f(t) \geqslant h_{2}>0, \quad h_{1}^{\prime} \geqslant g(t) \geqslant h_{2}^{\prime}>0 .
$$

Тогда для любого натурального $n, n>2[1 / a]-1$, где $a=h_{2}^{\prime} /\left(h_{1} e\right)$, выполнень неравенства

$$
\begin{aligned}
\mathbf{M}_{\tilde{V}}\left(H_{n}^{(f, g)}(\tilde{V})\right) & >\frac{h_{2}}{h_{1}^{\prime}}\left(\log _{2}(n+1)-\frac{1-\gamma_{n+1}}{\ln 2}-3 A_{n} \log _{2}(1+n)\right), \\
\mathbf{M}\left(P_{0} \log _{2} P_{0}\right) & >-\frac{h_{1}}{h_{2}^{\prime}}\left(\frac{\log _{2}(n+1)-1}{n+1}-3 A_{n}\left(\log _{2}\left(\frac{h_{1}}{h_{2}^{\prime}}\right)+1\right)\right), \\
\mathbf{M}\left(P_{0}\right) & \geqslant \frac{h_{1}}{h_{2}^{\prime}(n+1)},
\end{aligned}
$$

где

$$
b=\left(\left[\frac{1}{a}\right]+1\right)>1, \quad \gamma_{n}=\sum_{i=1}^{n} \frac{1}{i}-\ln n, \quad A_{n}=(1-a)^{n}(n+1)^{b} .
$$

Доказательство. Обозначим через $B_{n}$ событие $\left\{\Delta_{(n)} \leqslant h_{2}^{\prime} /\left(e h_{1}\right)\right\}$. При выполнении этого события, в силу леммы 1 , верны неравенства

$$
\begin{aligned}
\mathbf{M}\left(H\left(\frac{h_{2}}{h_{1}^{\prime}} \Delta_{0}, \ldots, \frac{h_{2}}{h_{1}^{\prime}} \Delta_{n}\right)\right) & \leqslant \mathbf{M}\left(H\left(p_{0}, \ldots, p_{n}\right)\right) \\
& \leqslant \mathbf{M}\left(H\left(\frac{h_{1}}{h_{2}^{\prime}} \Delta_{0}, \ldots, \frac{h_{1}}{h_{2}^{\prime}} \Delta_{0}\right)\right)
\end{aligned}
$$

В силу леммы 2 , при $n>2[1 / a]-1$, где $a=h_{2}^{\prime} /\left(h_{1} e\right)<1$, верно неравенство

$$
\mathbf{P}\left(B_{n}\right) \geqslant 1-(1-a)^{n}(n+1)^{b},
$$

где $b=([1 / a]+1)>1$.

Запишем математическое ожидание энтропии с помощью условных математических ожиданий

$$
\mathbf{M}\left(H\left(P_{0}, \ldots, P_{n}\right)\right)=\mathbf{M}\left(H\left(P_{0}, \ldots, P_{n}\right) \mid B_{n}\right) \mathbf{P}\left(B_{n}\right)+\mathbf{M}\left(H\left(P_{0}, \ldots, P_{n}\right) \mid \bar{B}_{n}\right) \mathbf{P}\left(\bar{B}_{n}\right) .
$$

Поскольку рассматривается энтропия - неотрицательная величина, можно отбросить второе слагаемое и получить неравенство

$$
\mathbf{M}\left(H\left(P_{0}, \ldots, P_{n}\right)\right) \geqslant \mathbf{M}\left(H\left(P_{0}, \ldots, P_{n}\right) \mid B_{n}\right) \mathbf{P}\left(B_{n}\right) .
$$


Используя лемму 1, можно продолжить цепочку неравенств:

$$
\mathbf{M}\left(H\left(P_{0}, \ldots, P_{n}\right) \mid B_{n}\right) \mathbf{P}\left(B_{n}\right) \geqslant \mathbf{M}\left(H\left(\frac{h_{2}}{h_{1}^{\prime}} \Delta_{0}, \ldots, \frac{h_{2}}{h_{1}^{\prime}} \Delta_{n}\right) \mid B_{n}\right) \mathbf{P}\left(B_{n}\right) .
$$

Для функции энтропии выполнено равенство

$$
H\left(c x_{1}, \ldots, c x_{n}\right)=c H\left(x_{1}, \ldots, x_{n}\right)-c \log _{2} c .
$$

Пользуясь этим равенством для $c=h_{2} / h_{1}^{\prime}$, получаем

$$
\mathbf{M}\left(H\left(\frac{h_{2}}{h_{1}^{\prime}} \Delta_{0}, \ldots, \frac{h_{2}}{h_{1}^{\prime}} \Delta_{n}\right) \mid B_{n}\right)=\frac{h_{2}}{h_{1}^{\prime}} \mathbf{M}\left(H\left(\Delta_{0}, \ldots, \Delta_{n}\right) \mid B_{n}\right)-\left(\frac{h_{2}}{h_{1}^{\prime}}\right) \log _{2}\left(\frac{h_{2}}{h_{1}^{\prime}}\right) .
$$

Выражение $-\left(h_{2} / h_{1}^{\prime}\right) \log _{2}\left(h_{2} / h_{1}^{\prime}\right)$ неотрицательно, поскольку $h_{2} / h_{1}^{\prime}<1$. Значит,

$$
\mathbf{M}\left(H\left(\frac{h_{2}}{h_{1}^{\prime}} \Delta_{0}, \ldots, \frac{h_{2}}{h_{1}^{\prime}} \Delta_{n}\right) \mid B_{n}\right) \geqslant \frac{h_{2}}{h_{1}^{\prime}} \mathbf{M}\left(H\left(\Delta_{0}, \ldots, \Delta_{n}\right) \mid B_{n}\right) .
$$

Рассмотрим величину $H\left(\Delta_{0}, \ldots, \Delta_{n}\right)$. Для сокращения записи формул, обозначим эту величину через $\xi_{n}$. Оценим модуль разности между условным математическим ожиданием и безусловным.

Рассмотрим разность $\mathbf{M}\left(\xi_{n}\right)-\mathbf{M}\left(\xi_{n} \mid B_{n}\right)$ и распишем математическое ожидание $\mathbf{M}\left(\xi_{n}\right)$ следующим образом:

$$
\mathbf{M}\left(\xi_{n}\right)-\mathbf{M}\left(\xi_{n} \mid B_{n}\right)=\mathbf{M}\left(\xi_{n} \mid B_{n}\right) \mathbf{P}\left(B_{n}\right)+\mathbf{M}\left(\xi_{n} \mid \bar{B}_{n}\right) \mathbf{P}\left(\bar{B}_{n}\right)-\mathbf{M}\left(\xi_{n} \mid B_{n}\right)
$$

Сгруппируем в правой части равенства выражения с множителем $\mathbf{M}\left(\xi_{n} \mid B_{n}\right)$ и вместо $\mathbf{P}\left(B_{n}\right)-1$ подставим $-\mathbf{P}\left(\bar{B}_{n}\right)$ :

$$
\mathbf{M}\left(\xi_{n}\right)-\mathbf{M}\left(\xi_{n} \mid B_{n}\right)=-\mathbf{M}\left(\xi_{n} \mid B_{n}\right) \mathbf{P}\left(\bar{B}_{n}\right)+\mathbf{M}\left(\xi_{n} \mid \bar{B}_{n}\right) \mathbf{P}\left(\bar{B}_{n}\right) .
$$

Величина $\xi_{n}$, как энтропия $(n+1)$ переменных, ограничена значением $\log _{2}(1+n)$. Модуль разности условного и безусловного математического ожидания оценим следующим образом:

$$
\left|\mathbf{M}\left(\xi_{n}\right)-\mathbf{M}\left(\xi_{n} \mid B_{n}\right)\right|<2 \log _{2}(1+n) \mathbf{P}\left(\bar{B}_{n}\right) .
$$

По лемме 2, при $n>2[1 / a]-1$, где $a=h_{2}^{\prime} /\left(h_{1} e\right)$, справедливо неравенство

$$
\mathbf{P}\left(\bar{B}_{n}\right) \leqslant(1-a)^{n}(n+1)^{b}, \quad a=h_{2}^{\prime} /\left(h_{1} e\right)<1, \quad b=([1 / a]+1)>1 .
$$

Поэтому при $n>2[1 / a]-1$ верно неравенство

$$
\left|\mathbf{M}\left(\xi_{n}\right)-\mathbf{M}\left(\xi_{n} \mid B_{n}\right)\right|<2 \log _{2}(1+n)(1-a)^{n}(n+1)^{b} .
$$

В [2] показано, что

$$
\mathbf{M}\left(H\left(\Delta_{0}, \ldots, \Delta_{n}\right)\right) \geqslant \log _{2}(n+1)-\frac{1-\gamma_{n+1}}{\ln 2},
$$

где

$$
\gamma_{n}=\sum_{i=1}^{n} \frac{1}{i}-\ln n
$$


Запишем математическое ожидание $\mathbf{M}\left(\xi_{n} \mid B_{n}\right)$ в виде

$$
\mathbf{M}\left(\xi_{n} \mid B_{n}\right)=\mathbf{M}\left(\xi_{n}\right)+\left(\mathbf{M}\left(\xi_{n} \mid B_{n}\right)-\mathbf{M}\left(\xi_{n}\right)\right) .
$$

Подставим оценки для разности условного и безусловного математического ожидания, и получим, что при $n>2[1 / a]-1$ верно неравенство

$$
\mathbf{M}\left(\xi_{n} \mid B_{n}\right)>\log _{2}(n+1)-\frac{1-\gamma_{n+1}}{\ln 2}-2 \log _{2}(1+n)(1-a)^{n}(n+1)^{b} .
$$

Ранее мы получили, что

$$
\mathbf{M} \xi_{n}>\frac{h_{2}}{h_{1}^{\prime}} \mathbf{M}\left(\xi_{n} \mid B_{n}\right) \mathbf{P}\left(B_{n}\right) .
$$

При $n>2[1 / a]-1$ справедливо неравенство

$$
\mathbf{P}\left(B_{n}\right) \geqslant 1-(1-a)^{n}(n+1)^{b},
$$

поэтому при $n>2[1 / a]-1$ верно неравенство

$$
\begin{array}{r}
\mathbf{M}\left(\xi_{n}\right)>\frac{h_{2}}{h_{1}^{\prime}}\left(\log _{2}(n+1)-\frac{1-\gamma_{n+1}}{\ln 2}-2 \log _{2}(1+n)(1-a)^{n}(n+1)^{b}\right) \\
\times\left(1-(1-a)^{n}(n+1)^{b}\right) .
\end{array}
$$

Обозначим для краткости выражение $(1-a)^{n}(n+1)^{b}$ через $A_{n}$, раскроем скобки в правой части неравенства и в качестве нижней оценки оставим только $\log _{2}(n+1)$ и слагаемые со знаком минус:

$$
\begin{aligned}
\left(\log _{2}(n+1)-\frac{1-\gamma_{n+1}}{\ln 2}-2 \log _{2}(1+n) A_{n}\right)\left(1-A_{n}\right) & \\
& \geqslant \log _{2}(n+1)-\frac{1-\gamma_{n+1}}{\ln 2}-3 \log _{2}(1+n) A_{n} .
\end{aligned}
$$

Из этого неравенства следует, что при $n>2[1 / a]-1$

$$
\mathbf{M}\left(H\left(P_{0}, \ldots, P_{n}\right)\right)>\frac{h_{2}}{h_{1}^{\prime}}\left(\log _{2}(n+1)-\frac{1-\gamma_{n+1}}{\ln 2}-3 \log _{2}(1+n) A_{n}\right) .
$$

Первое неравенство леммы доказано.

Рассмотрим математическое ожидание $\mathbf{M}\left(P_{0} \log _{2} P_{0}\right)$. Запишем его с помощью условных математических ожиданий:

$$
\mathbf{M}\left(P_{0} \log _{2} P_{0}\right)=\mathbf{M}\left(P_{0} \log _{2} P_{0} \mid B_{n}\right) \mathbf{P}\left(B_{n}\right)+\mathbf{M}\left(P_{0} \log _{2} P_{0} \mid \bar{B}_{n}\right) \mathbf{P}\left(\bar{B}_{n}\right) .
$$

Случайная величина $\left(P_{0} \log _{2} P_{0}\right)$ принимает значения от $-(1 / e) \log _{2} e$ до нуля. Учитывая это и принимая во внимание лемму 1 , получим неравенство

$$
\mathbf{M}\left(P_{0} \log _{2} P_{0}\right)>\mathbf{M}\left(\left(\frac{h_{1}}{h_{2}^{\prime}} \Delta_{0}\right) \log _{2}\left(\frac{h_{1}}{h_{2}^{\prime}} \Delta_{0}\right) \mid B_{n}\right) \mathbf{P}\left(B_{n}\right)-\left(\frac{1}{e}\right) \log _{2} e \mathbf{P}\left(\bar{B}_{n}\right) .
$$

Второе слагаемое в правой части неравенства $-(1 / e) \log _{2} e \mathbf{P}\left(\bar{B}_{n}\right)$ оценим снизу выражением $-\mathbf{P}\left(\bar{B}_{n}\right)$. Преобразуем условное математическое ожидание следующим образом:

$$
\mathbf{M}\left(\left(\frac{h_{1}}{h_{2}^{\prime}} \Delta_{0}\right) \log _{2}\left(\frac{h_{1}}{h_{2}^{\prime}} \Delta_{0}\right) \mid B_{n}\right)=\frac{h_{1}}{h_{2}^{\prime}} \mathbf{M}\left(\Delta_{0} \log _{2} \Delta_{0} \mid B_{n}\right)+\frac{h_{1}}{h_{2}^{\prime}} \log _{2}\left(\frac{h_{1}}{h_{2}^{\prime}}\right) \mathbf{M}\left(\Delta_{0} \mid \bar{B}_{n}\right) .
$$


Оценим разность между условным и безусловным математическим ожиданием для случайных величин $\Delta_{0}$ и $\Delta_{0} \log _{2} \Delta_{0}$ :

$$
\left.\mathbf{M}\left(\Delta_{0}\right) \mid B_{n}\right)=\left(\mathbf{M}\left(\Delta_{0} \mid \bar{B}_{n}\right)-\mathbf{M}\left(\Delta_{0} \mid B_{n}\right)\right) \mathbf{P}\left(\bar{B}_{n}\right),
$$

$\mathbf{M}\left(\Delta_{0} \log _{2} \Delta_{0}\right)-\mathbf{M}\left(\Delta_{0} \log _{2} \Delta_{0} \mid B_{n}\right)=\left(\mathbf{M}\left(\Delta_{0} \log _{2} \Delta_{0} \mid \bar{B}_{n}\right)-\mathbf{M}\left(\Delta_{0} \log _{2} \Delta_{0} \mid B_{n}\right)\right) \mathbf{P}\left(\bar{B}_{n}\right)$.

Значения случайных величин $\Delta_{0} \log _{2} \Delta_{0}$ и $\Delta_{0}$ ограничены по модулю единицей, поэтому верны неравенства

$$
\begin{aligned}
\left.\left|\mathbf{M}\left(\Delta_{0}\right)\right| \bar{B}_{n}\right) \mid & <\mathbf{P}\left(\bar{B}_{n}\right), \\
\left.\left|\mathbf{M}\left(\Delta_{0} \log _{2} \Delta_{0}\right)\right| \bar{B}_{n}\right) \mid & <\mathbf{P}\left(\bar{B}_{n}\right) .
\end{aligned}
$$

Согласно лемме 2, при $n>2[1 / a]-1$, где $a=h_{2}^{\prime} /\left(h_{1} e\right)$, справедливо неравенство

$$
\mathbf{P}\left(\bar{B}_{n}\right)<(1-a)^{n}(n+1)^{b},
$$

где $b=([1 / a]+1)>1$. Выражение $(1-a)^{n}(n+1)^{b}$ мы выше обозначили через $A_{n}$. При $n>2[1 / a]-1$ выполнены неравенства

$$
\begin{aligned}
\left.\left|\mathbf{M}\left(\Delta_{0}\right)\right| B_{n}\right) \mid & <2 A_{n}, \\
\left|\mathbf{M}\left(\Delta_{0} \log _{2} \Delta_{0}\right)-\mathbf{M}\left(\Delta_{0} \log _{2} \Delta_{0} \mid B_{n}\right)\right| & <2 A_{n} .
\end{aligned}
$$

В [2] показано, что

$$
\begin{aligned}
\mathbf{M}\left(\Delta_{0} \log _{2} \Delta_{0}\right) & =-\frac{1}{n+1}\left(\log _{2}(n+1)-\frac{1-\gamma_{n+1}}{\ln 2}\right), \\
\mathbf{M}\left(\Delta_{0}\right) & =\frac{1}{n+1} .
\end{aligned}
$$

где

$$
\gamma_{n}=\sum_{i=1}^{n} \frac{1}{i}-\ln n .
$$

Выражение $\left(1-\gamma_{n+1}\right) / \ln 2$ всегда меньше единицы. Значения условных математических ожиданий отличаются от безусловных на величину по модулю, меньшую $2 A_{n}$, поэтому при $n>2[1 / a]-1$ верны неравенства

$$
\begin{gathered}
\mathbf{M}\left(\Delta_{0} \log _{2} \Delta_{0} \mid B_{n}\right) \geqslant-\frac{\log _{2}(n+1)-1}{n+1}-2 A_{n}, \\
\mathbf{M}\left(\Delta_{0} \mid B_{n}\right) \geqslant \frac{1}{n+1}-2 A_{n} .
\end{gathered}
$$

Собирая полученные оценки

$$
\begin{aligned}
\mathbf{M}\left(P_{0} \log _{2} P_{0}\right)>\left(\frac{h_{1}}{h_{2}^{\prime}} \mathbf{M}\left(\Delta_{0} \log _{2} \Delta_{0} \mid B_{n}\right)\right) \mathbf{P}\left(B_{n}\right) & \\
& +\left(\frac{h_{1}}{h_{2}^{\prime}} \log _{2}\left(\frac{h_{1}}{h_{2}^{\prime}}\right) \mathbf{M}\left(\Delta_{0} \mid B_{n}\right)\right) \mathbf{P}\left(B_{n}\right)-\mathbf{P}\left(\bar{B}_{n}\right),
\end{aligned}
$$


получаем, что при $n>2[1 / a]-1$ справедливо неравенство

$$
\begin{aligned}
\mathbf{M}\left(P_{0} \log _{2} P_{0}\right)>\left(1-A_{n}\right) \frac{h_{1}}{h_{2}^{\prime}}\left(-\frac{\log _{2}(n+1)-1}{n+1}-2 A_{n}\right) & \\
& +\left(1-A_{n}\right)\left(\frac{h_{1}}{h_{2}^{\prime}} \log _{2}\left(\frac{h_{1}}{h_{2}^{\prime}}\right)\right)\left(\frac{1}{n+1}-2 A_{n}\right)-A_{n} .
\end{aligned}
$$

Упрощая правую часть неравенства, получаем, что при $n>2[1 / a]-1$

$$
\mathbf{M}\left(P_{0} \log _{2} P_{0}\right)>-\frac{h_{1}}{h_{2}^{\prime}} \frac{\log _{2}(n+1)-1}{n+1}-3 A_{n} \frac{h_{1}}{h_{2}^{\prime}}\left(1+\log _{2}\left(\frac{h_{1}}{h_{2}^{\prime}}\right)\right) .
$$

Второе неравенство леммы доказано.

Рассмотрим математическое ожидание $M\left(P_{0}\right)$. В силу леммы 1

$$
\mathbf{M}\left(P_{0}\right) \geqslant \frac{h_{1}}{h_{2}^{\prime}} \mathbf{M}\left(\Delta_{0}\right) \text {. }
$$

Поскольку

$$
\mathbf{M}\left(\Delta_{0}\right)=\frac{1}{n+1}
$$

получаем требуемое неравенство

$$
\mathbf{M}\left(P_{0}\right) \geqslant \frac{h_{1}}{h_{2}^{\prime}} \frac{1}{n+1} .
$$

Доказательство леммы закончено.

Для оценивания математического ожидания нижней энтропийной оценки нам понадобится следующая лемма.

Лемма 4. Пусть $r \in(0,1),\left(\begin{array}{l}n \\ j\end{array}\right)-$ биномиальныци коэффициент, тогда для любого $n \in \mathbf{N}$

$$
\sum_{j \in\left[\gamma_{n}, \delta_{n}\right]}\left(\begin{array}{l}
n \\
j
\end{array}\right) r^{j}(1-r)^{n-j}>1-2 \frac{C\left(1-2 r+2 r^{2}\right)}{\sqrt{r(1-r) n}}-\frac{1}{\sqrt{2 \pi}} e^{-\left(n^{1 / 3}\right) /(2 r(1-r))} \frac{\sqrt{r(1-r)}}{n^{1 / 6}},
$$

гेe

$$
\gamma_{n}=\left[r n-n^{2 / 3}\right], \quad \delta_{n}=\left[r n+n^{2 / 3}\right],
$$

C - абсолютная постоянная.

Доказательство. Пусть $\xi_{i}, i=1, \ldots, n,-$ независимые случайные величины, имеющие распределение Бернулли с вероятностью успеха $r$. Обозначим

$$
S_{n}=\sum_{i=1}^{n} \xi_{i}
$$

Интересующее нас выражение можно записать в виде

$$
\sum_{j \in\left[\gamma_{n}, \delta_{n}\right]}\left(\begin{array}{l}
n \\
j
\end{array}\right) r^{j}(1-r)^{n-j}=\mathbf{P}\left(r n-n^{2 / 3}-1<S_{n} \leqslant r n+n^{2 / 3}\right) .
$$


Преобразуем последнее выражение так, чтобы было удобно применить интегральную предельную теорему Муавра-Лапласа:

$$
\mathbf{P}\left(r n-n^{2 / 3}-1<S_{n} \leqslant r n+n^{2 / 3}\right)=\mathbf{P}\left(\frac{-n^{2 / 3}-1}{\sqrt{r(1-r) n}}<\frac{S_{n}-r n}{\sqrt{r(1-r) n}} \leqslant \frac{n^{2 / 3}}{\sqrt{r(1-r) n}}\right) .
$$

Положим

$$
a_{n}^{*}=\frac{-n^{2 / 3}-1}{\sqrt{r(1-r) n}}, \quad b_{n}^{*}=\frac{n^{2 / 3}}{\sqrt{r(1-r) n}}, \quad S_{n}^{*}=\frac{S_{n}-r n}{\sqrt{r(1-r) n}} .
$$

Заметим, что при $n \rightarrow \infty$ величины $a_{n}^{*} \rightarrow-\infty, b_{n}^{*} \rightarrow-\infty$, поэтому при $n \rightarrow \infty$

$$
\mathbf{P}\left(a_{n}^{*}<\frac{S_{n}-r n}{\sqrt{r(1-r) n}} \leqslant b_{n}^{*}\right) \rightarrow 1 .
$$

Оценим скорость сходимости. Пусть $F_{n}-$ функция распределения случайной величины $S_{n}^{*}$ и $\Phi(x)$ - функция распределения стандартного нормального распределения. Поскольку $\mathbf{M}\left(\xi_{1}^{3}\right)=r$, то выполнено условие теоремы Берри-Эссеена, и при всех $n \in \mathbf{N}$ верно неравенство

$$
\sup _{x}\left|F_{n}(x)-\Phi(x)\right|<\frac{C \mathbf{M}\left|\xi_{1}-r\right|^{3}}{\sqrt{r^{3}(1-r)^{3} n}},
$$

где $C$ - абсолютная постоянная. Поскольку

$$
\mathbf{M}\left|\xi_{1}-r\right|^{3}=r(1-r)\left(1-2 r+2 r^{2}\right),
$$

то при всех $n \in \mathbf{N}$ верно неравенство

$$
\sup _{x}\left|F_{n}(x)-\Phi(x)\right|<\frac{C\left(1-2 r+2 r^{2}\right)}{\sqrt{r(1-r) n}} .
$$

Представим

$$
\mathbf{P}\left(a_{n}^{*}<\frac{\xi_{n}-r n}{\sqrt{r(1-r) n}} \leqslant b_{n}^{*}\right)=F_{n}\left(b_{n}^{*}\right)-F_{n}\left(a_{n}^{*}\right) .
$$

Затем прибавим и отнимем величины $\Phi\left(a_{n}^{*}\right)$ и $\Phi\left(b_{n}^{*}\right)$, и получим

$$
F_{n}\left(b_{n}^{*}\right)-F_{n}\left(a_{n}^{*}\right)=\Phi\left(b_{n}^{*}\right)-\Phi\left(a_{n}^{*}\right)+\left(F_{n}\left(b_{n}^{*}\right)-\Phi\left(b_{n}^{*}\right)\right)-\left(F_{n}\left(a_{n}^{*}\right)-\Phi\left(a_{n}^{*}\right)\right) .
$$

Из этого равенства получаем нижнюю оценку

$$
F_{n}\left(b_{n}^{*}\right)-F_{n}\left(a_{n}^{*}\right)>\Phi\left(b_{n}^{*}\right)-\Phi\left(a_{n}^{*}\right)-\left|F_{n}\left(b_{n}^{*}\right)-\Phi\left(b_{n}^{*}\right)\right|-\left|F_{n}\left(a_{n}^{*}\right)-\Phi\left(a_{n}^{*}\right)\right| .
$$

Выражение $\Phi\left(b_{n}^{*}\right)-\Phi\left(a_{n}^{*}\right)$ можно переписать в виде

$$
\left(\Phi\left(b_{n}^{*}\right)-\Phi\left(a_{n}^{*}\right)\right)=1+\left(\Phi\left(b_{n}^{*}\right)-1-\Phi\left(a_{n}^{*}\right)\right) .
$$

Оценим выражение $\Phi\left(b_{n}^{*}\right)-1-\Phi\left(a_{n}^{*}\right)$. Известно [4], что при $x>0$

$$
\frac{1}{\sqrt{2 \pi}} e^{-x^{2} / 2} \frac{1}{x}\left(1-\frac{1}{x^{2}}\right)<1-\Phi(x)<\frac{1}{\sqrt{2 \pi}} e^{-x^{2} / 2} \frac{1}{x} .
$$


При

$$
x=b_{n}^{*}=\frac{n^{2 / 3}}{\sqrt{r(1-r) n}}=\frac{n^{1 / 6}}{\sqrt{r(1-r)}},
$$

справедливо неравенство

$$
-\left(1-\Phi\left(b_{n}^{*}\right)\right)>-\frac{1}{\sqrt{2 \pi}} e^{-n^{1 / 3} /(2 r(1-r))} \frac{\sqrt{r(1-r)}}{n^{1 / 6}} .
$$

При

$$
x=a_{n}^{*}=-\frac{n^{2 / 3}+1}{\sqrt{r(1-r) n}}
$$

справедливы соотношения

$$
\begin{aligned}
-\Phi\left(a_{n}^{*}\right)=1-\Phi\left(-a_{n}^{*}\right)> & \frac{1}{\sqrt{2 \pi}} e^{-2 n^{1 / 3} /(r(1-r)} \frac{\sqrt{r(1-r) n}}{n^{2 / 3}+1}\left(1-\frac{r(1-r) n}{\left(n^{2 / 3}+1\right)^{2}}\right), \\
\Phi\left(b_{n}^{*}\right)-1-\Phi\left(a_{n}^{*}\right)>- & \frac{1}{\sqrt{2 \pi}} e^{-2 n^{1 / 3} /\left(r(1-r) \frac{\sqrt{r(1-r)}}{n^{1 / 6}}\right.} \\
& \times\left(1-\frac{e^{-4}}{n^{2 / 3}+1}\left(1-\frac{r(1-r) n}{\left(n^{2 / 3}+1\right)^{2}}\right)\right) .
\end{aligned}
$$

Выражение под скобками положительно и меньше единицы, поэтому

$$
\Phi\left(b_{n}^{*}\right)-1-\Phi\left(a_{n}^{*}\right)>-\frac{1}{\sqrt{2 \pi}} e^{-n^{1 / 3} /(2 r(1-r))} \frac{\sqrt{r(1-r)}}{n^{1 / 6}} .
$$

Отсюда следует, что

$$
F_{n}\left(b_{n}^{*}\right)-F_{n}\left(a_{n}^{*}\right)>1-2 \frac{C\left(1-2 r+2 r^{2}\right)}{\sqrt{r(1-r) n}}-\frac{1}{\sqrt{2 \pi}} e^{-n^{1 / 3} /(2 r(1-r))} \frac{\sqrt{r(1-r)}}{n^{1 / 6}} .
$$

Доказательство леммы закончено.

Лемма 5. Пусть функции плотности $f$ и $g$ удовлетворяют следующим ограничениям: найдутся $(a, b) \subset(0,1), a \neq b$, такие, что для произвольно выбранного $x \in(a, b)$ выполняются неравенства

$$
c_{1}>f(x)>c_{2}>0, \quad c_{1}^{\prime}>g(x)>c_{2}^{\prime}>0 .
$$

Тогда для любого $\varepsilon>0$ существует такое натуральное $n_{0}$, что для любого $n>n_{0}$

$$
\mathbf{M}_{\widetilde{V}}\left(H_{n}^{(f, g)}(\tilde{V})\right) \geqslant \frac{c_{2} r}{c_{1}^{\prime}} \log _{2}(r n+1)-\frac{c_{2} r}{c_{1}^{\prime}} \frac{1-\gamma}{\ln 2}-\varepsilon,
$$

2วe

$$
r=\int_{a}^{b} g(x) d x
$$

$\gamma$ - постоянная Эйлера. 
Доказательство. Обозначим через $E_{j}, j=0,1, \ldots, n$, событие, состоящее в том что ровно $j$ записей библиотеки находятся в интервале $(a, b)$. Справедливо равенство

$$
\mathbf{M}_{\tilde{V}}\left(H_{n}^{(f, g)}(\tilde{V})\right)=\sum_{j=0}^{n} \mathbf{M}_{\tilde{V}}\left(H_{n}^{(f, g)}(\tilde{V}) \mid E_{j}\right) \mathbf{P}\left(E_{j}\right) .
$$

Поскольку функция энтропии неотрицательна по определению, для оценивания снизу можно рассмотреть лишь некоторые слагаемые:

$$
\mathbf{M}_{\widetilde{V}}\left(H_{n}^{(f, g)}(\tilde{V})\right) \geqslant \sum_{j=\left[r n-n^{2 / 3}\right]}^{\left[r n+n^{2 / 3}\right]} \mathbf{M}_{\widetilde{V}}\left(H_{n}^{(f, g)}(\tilde{V}) \mid E_{j}\right) \mathbf{P}\left(E_{j}\right),
$$

где, по условию леммы,

$$
r=\int_{a}^{b} g(x) d x .
$$

Оценим значение $\mathbf{M}_{\tilde{V}}\left(H_{n}^{(f, g)}(\widetilde{V}) \mid E_{j}\right)$. Записи библиотеки, которые попали в интервал $(a, b)$, обозначим через $Y_{1}^{\prime}, \ldots, Y_{j}^{\prime}$. Точки $a$ и $b$ обозначим через $Y_{(0)}$ и $Y_{(j+1)}$ соответственно. Вероятности попадания запроса между этими записями обозначим через $P_{i}^{\prime}=\mathbf{P}\left(x \in\left(Y_{(i)}^{\prime}, Y_{(i+1)}^{\prime}\right)\right), i=0,1, \ldots, j$. Справедливо неравенство

$$
H_{n}^{(f, g)}(\widetilde{V})>\sum_{i=1}^{j-1}-P_{i}^{\prime} \log _{2} P_{i}^{\prime} .
$$

Случайные величины $Y_{i}^{\prime}$ имеют функцию плотности $(1 / r) g(t)$.

Вероятность $P_{i}^{\prime}$ запишем следующим образом:

$$
P_{i}^{\prime}=\mathbf{P}\left(x \in\left(Y_{(i)}^{\prime}, Y_{(i+1)}^{\prime}\right)=\mathbf{P}(x \in(a, b)) \mathbf{P}\left(x \in\left(Y_{(i)}^{\prime}, Y_{(i+1)}^{\prime}\right) \mid x \in(a, b)\right) .\right.
$$

Обозначим условную вероятность в правой части равенства через $P_{i}^{\prime \prime}$ и выразим через нее сумму $\sum_{i=1}^{j-1}-P_{i}^{\prime} \log _{2} P_{i}^{\prime}$ :

$$
\begin{aligned}
\sum_{i=1}^{j-1}-P_{i}^{\prime} \log _{2} P_{i}^{\prime}=\mathbf{P}(x \in(a, b))\left(\sum_{i=1}^{j-1}-P_{i}^{\prime \prime} \log _{2} P_{i}^{\prime \prime}\right) \\
-\mathbf{P}(x \in(a, b)) \log _{2} \mathbf{P}(x \in(a, b))\left(\sum_{i=1}^{j-1} P_{i}^{\prime \prime}\right) .
\end{aligned}
$$

Последнее слагаемое в правой части неравенства отрицательно. Для получения нижней оценки его можно опустить:

$$
\sum_{i=1}^{j-1}-P_{i}^{\prime} \log _{2} P_{i}^{\prime} \geqslant \mathbf{P}(x \in(a, b))\left(\sum_{i=1}^{j-1}-P_{i}^{\prime \prime} \log _{2} P_{i}^{\prime \prime}\right) .
$$

Обозначим через $X^{\prime \prime}$ случайную величину, принимающую значение в интервале $(a, b)$ и имеющую функцию плотности $(1 / d) f(t)$, где $d=\mathbf{P}(x \in(a, b))$. Через $Y_{i}^{\prime \prime}, i=1, \ldots, j$, обозначим независимые случайные величины, принимающие значение в интервале $(a, b)$ 
и имеющие функцию плотности $(1 / r) g(t)$. Случайную величину $\mathbf{P}\left(X^{\prime \prime} \in\left(Y_{(i)}^{\prime \prime}, Y_{(i+1)}^{\prime \prime}\right)\right)$ при $i$, принимающем значения от 1 до $j-1$, обозначим через $P_{i}^{\prime \prime}$. С помощью этих случайных величин условное математическое ожидание для случайной величины $H_{n}^{(f, g)}(\tilde{V})$ можно оценить следующим образом:

$$
\mathbf{M}_{\tilde{V}}\left(H_{n}^{(f, g)}(\tilde{V}) \mid E_{j}\right) \geqslant d \mathbf{M}\left(\sum_{i=1}^{j-1}-P_{i}^{\prime \prime} \log _{2} P_{i}^{\prime \prime}\right) .
$$

Преобразованием вида

$$
v=\frac{(\xi-a)}{b-a}
$$

перейдем от случайных величин, принимающих значение из интервала $(a, b)$, к случайным величинам, принимающим значения из интервала $(0,1)$. Тогда случайная величина $X^{\prime \prime}$ преобразуется в случайную величину $X^{*}$ с функцией плотности

$$
f^{*}(t)=\frac{(b-a)}{d} f((b-a) t+a) .
$$

Случайная величина $Y_{i}^{\prime \prime}$ преобразуется в случайную величину $Y_{i}^{*}$ с функцией плотности

$$
g^{*}(t)=\frac{(b-a)}{r} g((b-a) t+a) .
$$

А для случайных величин $P_{i}^{*}=\mathbf{P}\left(X^{*} \in\left(Y_{(i)}^{*}, Y_{(i+1)}^{*}\right)\right)$ оценки переписываются следующим образом:

$$
\mathbf{M}_{\widetilde{V}}\left(H_{n}^{(f, g)}(\tilde{V}) \mid E_{j}\right) \geqslant d \mathbf{M}\left(\sum_{i=1}^{j-1}-P_{i}^{*} \log _{2} P_{i}^{*}\right) .
$$

Для функций плотности $f^{*}$ и $g^{*}$ при всех $t \in(0,1)$ справедливы неравенства

$$
\begin{aligned}
& \frac{c_{1}(b-a)}{d}>f^{*}(x)>\frac{c_{2}(b-a)}{d}>0, \\
& \frac{c_{1}^{\prime}(b-a)}{r}>g^{*}(x)>\frac{c_{2}^{\prime}(b-a)}{r}>0 .
\end{aligned}
$$

Рассмотрим случайную библиотеку $\tilde{V}^{*}=\left(Y_{1}^{*}, \ldots, Y_{j}^{*}\right)$. По лемме 3, при $j$, большем чем 2[1/a] -1 , где $a=\left(c_{2}^{\prime} d\right) /\left(c_{r} e\right)$, выполнены следующие неравенства:

$$
\begin{gathered}
\mathbf{M}_{\widetilde{V}^{*}}\left(H_{j}^{\left(f^{*}, g^{*}\right)}\left(\tilde{V}^{*}\right)\right)>\frac{c_{2} r}{c_{1}^{\prime} d} \log _{2}(j+1)-\frac{c_{2} r}{c_{1}^{\prime} d} \frac{1-\gamma_{j+1}}{\ln 2}-3 \frac{c_{2} r}{c_{1}^{\prime} d} \log _{2}(1+j) A_{j}, \\
\mathbf{M}\left(P_{0}^{*} \log _{2} P_{0}^{*}\right)>-\frac{c_{1} r}{c_{2}^{\prime} d} \frac{\log _{2}(j+1)-1}{j+1}-3 A_{j} \frac{c_{1} r}{c_{2}^{\prime} d}\left(1+\log _{2}\left(\frac{c_{1} r}{c_{2}^{\prime} d}\right)\right),
\end{gathered}
$$

где

$$
b=\left(\left[\frac{1}{a}\right]+1\right)>1, \quad \gamma_{j}=\sum_{i=1}^{j} \frac{1}{i}-\ln j, \quad A_{j}=(1-a)^{j}(j+1)^{b} .
$$

Представим сумму $\sum_{i=1}^{j-1}-P_{i}^{*} \log _{2} P_{i}^{*}$ в виде

$$
\sum_{i=1}^{j-1}-P_{i}^{*} \log _{2} P_{i}^{*}=H_{j}^{\left(f^{*}, g^{*}\right)}\left(\tilde{V}^{*}\right)+P_{0}^{*} \log _{2} P^{*} 0+P_{j}^{*} \log _{2} P_{j}^{*} .
$$


Получаем, что

$$
\begin{aligned}
\mathbf{M}_{\tilde{V}}\left(H_{n}^{(f, g)}(\tilde{V}) \mid E_{j}\right) \geqslant \frac{c_{2} r}{c_{1}^{\prime}} & \log _{2}(j+1)-\frac{c_{2} r}{c_{1}^{\prime}} \frac{1-\gamma_{j+1}}{\ln 2} \\
& -3 \frac{c_{2} r}{c_{1}^{\prime}} \log _{2}(1+j) A_{j}-\frac{2 c_{1} r}{c_{2}^{\prime}} \frac{\log _{2}(j+1)-1}{j+1} \\
& -6 A_{j} \frac{c_{1} r}{c_{2}^{\prime}}\left(1+\log _{2}\left(\frac{c_{1} r}{c_{2}^{\prime} d}\right)\right) .
\end{aligned}
$$

Значения $j$ меняются в пределах от $\left[r n-n^{2 / 3}\right]$ до $\left[r n+n^{2 / 3}\right]$, поэтому при $n \rightarrow \infty$ индекс $j \rightarrow \infty$. В неравенстве 1 все слагаемые в левой части, кроме $\left(c_{2} r /\left(c_{1}^{\prime} d\right)\right) \log _{2}(j+1)$ и $\left(c_{2} r /\left(c_{1}^{\prime} d\right)\right)\left(1-\gamma_{j+1}\right) / \ln 2$, стремятся к нулю при $j \rightarrow \infty$, в то время как

$$
\frac{c_{2} r}{c_{1}^{\prime} d} \frac{1-\gamma_{j+1}}{\ln 2}=\frac{c_{2} r}{c_{1}^{\prime} d} \frac{1-\gamma}{\ln 2}+\frac{c_{2} r}{c_{1}^{\prime} d} \frac{\gamma-\gamma_{j+1}}{\ln 2}
$$

Второе слагаемое в правой части равенства стремится к нулю при $j \rightarrow \infty$.

Для любого положительного $\varepsilon$ существует такое натуральное $n_{0}$, что при любом $n>n_{0}$ и при любом значении индекса $j$ в пределах от $\left[r n-n^{2 / 3}\right]$ до $\left[r n+n^{2 / 3}\right]$ справедливо неравенство

$$
\mathbf{M}_{\widetilde{V}}\left(H_{n}^{(f, g)}(\tilde{V}) \mid E_{j}\right) \geqslant \frac{c_{2} r}{c_{1}^{\prime}} \log _{2}(j+1)-\frac{c_{2} r}{c_{1}^{\prime}} \frac{1-\gamma}{\ln 2}-\varepsilon .
$$

Подставив получившиеся оценки в неравенство для $\mathbf{M}_{\tilde{V}}\left(H_{n}^{(f, g)}(\tilde{V})\right)$, получим, что для любого положительного вещественного $\varepsilon$ существует такое натуральное $n_{0}$, что для любого $n>n_{0}$

$$
\mathbf{M}_{\widetilde{V}}\left(H_{n}^{(f, g)}(\tilde{V})\right) \geqslant \sum_{j=\left[r n-n^{2 / 3}\right]}^{\left[r n+n^{2 / 3}\right]}\left(\frac{c_{2} r}{c_{1}^{\prime}} \log _{2}(j+1)-\frac{c_{2} r}{c_{1}^{\prime}} \frac{1-\gamma}{\ln 2}-\varepsilon\right) \mathbf{P}\left(E_{j}\right) .
$$

Далее для сокращения записи формул не будем указывать пределы суммирования. Раскроем скобки в правой части неравенства и вынесем за пределы суммирования множители, не зависящие от $j$ :

$$
\begin{aligned}
\sum_{j}\left(\frac{c_{2} r}{c_{1}^{\prime} d} \log _{2}(j+1)\right. & \left.-\frac{c_{2}}{c_{1}^{\prime}} \frac{1-\gamma}{\ln 2}-\varepsilon\right) \mathbf{P}\left(E_{j}\right) \\
= & \frac{c_{2} r}{c_{1}^{\prime}} \sum_{j} \log _{2}(j+1) \mathbf{P}\left(E_{j}\right)-\frac{c_{2} r}{c_{1}^{\prime}} \frac{1-\gamma}{\ln 2} \sum_{j} \mathbf{P}\left(E_{j}\right)-\varepsilon \sum_{j} \mathbf{P}\left(E_{j}\right) .
\end{aligned}
$$

В слагаемых со знаком минус сумму $\sum_{j} \mathbf{P}\left(E_{j}\right)$ оценим единицей. Так как функция $\log _{2}(j+1)$ монотонно возрастает, оценим ее снизу величиной $\log _{2}\left(\left[r n-n^{2 / 3}\right]\right)$. Получим, что

$$
\begin{aligned}
\frac{c_{2} r}{c_{1}^{\prime}} \sum_{j} \log _{2}(j+1) \mathbf{P}\left(E_{j}\right)-\frac{c_{2} r}{c_{1}^{\prime}} \frac{1-\gamma}{\ln 2} \sum_{j} \mathbf{P}\left(E_{j}\right)-\varepsilon \sum_{j} \mathbf{P}\left(E_{j}\right) \\
\qquad \frac{c_{2} r}{c_{1}^{\prime}} \log _{2}\left(\left[r n-n^{2 / 3}\right]\right) \sum_{j} \mathbf{P}\left(E_{j}\right)-\frac{c_{2} r}{c_{1}^{\prime}} \frac{1-\gamma}{\ln 2}-\varepsilon .
\end{aligned}
$$


По лемме 4, выражение $\sum_{j} \mathbf{P}\left(E_{j}\right)$ отличается от единицы на величину, которая при $n \rightarrow \infty$ есть $O\left(1 /\left(n^{1 / 6}\right)\right)$. Выражение $\log _{2}\left(\left[r n-n^{2 / 3}\right]\right)$ также отличается от $\log _{2}(r n+1)$ на бесконечно малую величину при $n \rightarrow \infty$. Учитывая это, получаем, что для любого вещественного положительного $\varepsilon$ существует такое натуральное $n_{0}$, что для любого $n>n_{0}$ выполнено неравенство

$$
\mathbf{M}_{\tilde{V}}\left(H_{n}^{(f, g)}(\tilde{V})\right) \geqslant \frac{c_{2} r}{c_{1}^{\prime}} \log _{2}(r n+1)-\frac{c_{2} r}{c_{1}^{\prime}} \frac{1-\gamma}{\ln 2}-\varepsilon .
$$

Доказательство леммы закончено.

Доказательство теоремь 1. В случае, когда $s=1$, утверждение теоремы верно в силу леммы 5.

Рассмотрим случай, когда известно $s, s>1$, интервалов, на которых функции плотности $f$ и $g$ ограничены и отделены от нуля. Поскольку, по условию теоремы, интервалы не пересекаются, функцию энтропии случайных величин $P_{0}, \ldots, P_{n}$

$$
H\left(P_{0}, \ldots, P_{n}\right)=\sum_{j=0}^{n} P_{j} \log _{2} P_{j}
$$

можно разбить на $s$ слагаемых, где слагаемое с номером $i, i=1, \ldots, s$, зависит только от вероятностей тех интервалов $\left(Y_{(j)}, Y_{(j+1)}\right)$, которые полностью лежат в интервале $\left(a_{i}, b_{i}\right)$.

При доказательстве леммы 5 мы показали, что для любого положительного вещественного $\varepsilon$ существует такое натуральное $n_{0}$, что для любого натурального $n$, большего $n_{0}$, математическое ожидание слагаемого с номером $i$ можно оценить величиной

$$
\frac{e_{2}^{i} r_{i}}{k_{1}^{i}} \log _{2}\left(r_{i} n+1\right)-\frac{e_{2}^{i} r_{i}}{k_{1}^{i}} \frac{1-\gamma}{\ln 2}-\varepsilon .
$$

Суммируя эти оценки, получаем, что для любого положительного вещественного $\varepsilon$ существует такое натуральное $n_{0}$, что для любого натурального $n$, большего $n_{0}$,

$$
\mathbf{M}_{\tilde{V}}\left(T_{n}^{(f, g)}(\tilde{V})\right) \geqslant \sum_{i=1}^{s} \frac{e_{2}^{i} r_{i}}{k_{1}^{i}} \log _{2}\left(r_{i} n+1\right)-\frac{1-\gamma}{\ln 2} \sum_{i=1}^{s} \frac{e_{2}^{i} r_{i}}{k_{1}^{i}}-\varepsilon .
$$

Доказательство теоремы закончено.

\section{4. Доказательство теоремы 2}

Для доказательства теоремы нам понадобится следующая лемма. Эта лемма соответствует следствию 2.

Лемма 6. Пусть функиии плотности $f$ и $g$ интегрируемы по Риману и удовлетворяют следующим условиям: для произвольного $x \in(0,1)$

$$
c_{1} \geqslant f(x) \geqslant c_{2}>0, \quad c_{1}^{\prime} \geqslant g(x) \geqslant c_{2}^{\prime}>0 .
$$

Тогда при $n \rightarrow \infty$

$$
\mathbf{M}_{\widetilde{V}}\left(T_{n}^{(f, g)}(\tilde{V})\right) \sim \log _{2} n
$$


Доказательство. Любую задачу поиска идентичных объектов можно решить с помощью бинарного поиска. Сложность бинарного поиска зависит только от мощности библиотеки и не превосходит $\log _{2}(n+1)$. Поэтому оцениваемая величина $\mathbf{M}_{\widetilde{V}}\left(T_{n}^{(f, g)}(\tilde{V})\right)$ при увеличении $n$ асимптотически не больше $\log _{2} n$. Покажем, что эта величина также асимптотически не меньше $\log _{2} n$.

Разобьем интервал $(0,1)$ на $\left[\log _{2} n\right]$ одинаковых интервалов $\left(a_{j}, b_{j}\right)$, где

$$
a_{j}=\frac{j-1}{\left[\log _{2} n\right]}, \quad b_{j}=\frac{j}{\left[\log _{2} n\right]}, \quad j=1, \ldots,\left[\log _{2} n\right] .
$$

Введем обозначения для инфимума и супремума функций $f$ и $g$ на каждом интервале, полагая

$$
\begin{aligned}
& f_{\text {sup }}^{j}=\sup _{t \in\left(a_{j}, b_{j}\right)} f(t), \quad f_{\text {inf }}^{j}=\inf _{t \in\left(a_{j}, b_{j}\right)} f(t), \quad j=1, \ldots,\left[\log _{2} n\right], \\
& g_{\text {sup }}^{j}=\sup _{t \in\left(a_{j}, b_{j}\right)} g(t), \quad g_{\text {inf }}^{j}=\inf _{t \in\left(a_{j}, b_{j}\right)} g(t), \quad j=1, \ldots,\left[\log _{2} n\right] .
\end{aligned}
$$

Обозначим через $B_{i_{j}}, 0 \leqslant i_{j} \leqslant n, 1 \leqslant j \leqslant\left[\log _{2} n\right]$, событие, состоящее в том, что в интервале $\left(a_{j}, b_{j}\right)$ ровно $i_{j}$ записей библиотеки. Обозначим через $r_{j}$ интеграл функции $g$ по $\left(a_{j}, b_{j}\right)$, через $d_{j}$ - интеграл функции $f$ по $\left(a_{j}, b_{j}\right)$. Через $J_{j}$ обозначим множество значений индекса $i_{j}$, которые находятся в пределах от $r_{j} n-n^{2 / 3}$ до $r_{j} n+n^{2 / 3}$.

Поскольку функция энтропии принимает неотрицательные значения, можно записать, что

$$
\begin{aligned}
\mathbf{M}_{\tilde{V}}\left(H_{n}^{(f, g)}(\tilde{V})\right) \geqslant \sum_{J_{0}} \mathbf{M}_{\tilde{V}}\left(H_{n}^{(f, g)}(\tilde{V}) \mid\right. & \left.B_{i_{0}}\right) \mathbf{P}\left(B_{i_{0}}\right)+\ldots \\
& +\sum_{J_{\left[\log _{2} n\right]}} \mathbf{M}_{\tilde{V}}\left(H_{n}^{(f, g)}(\tilde{V}) \mid B_{\left.i_{\left[\log _{2} n\right]}\right)}\right) \mathbf{P}\left(B_{i_{\left[\log _{2} n\right]}}\right) .
\end{aligned}
$$

Рассмотрим слагаемое, которое соответствует интервалу $\left(a_{j}, b_{j}\right)$. При доказательстве леммы 5 было доказано неравенство (1), которое применительно к условному математическому ожиданию для события $B_{i_{j}}$ записывается следующим образом: при $i_{j}$ большем, чем $2\left[1 / a_{j}\right]-1$, где

$$
a_{j}=\frac{g_{\text {inf }}^{j} d_{j}}{f_{\text {sup }}^{j} r_{j} e},
$$

верно неравенство

$$
\begin{aligned}
\mathbf{M}_{\tilde{V}}\left(H_{n}^{(f, g)}(\tilde{V}) \mid B_{i_{j}}\right) \geqslant & \frac{f_{\text {inf }}^{j} r_{j}}{g_{\text {sup }}^{j}} \log _{2}\left(i_{j}+1\right)-\frac{f_{\text {inf }}^{j} r_{j}}{g_{\text {sup }}^{j}} \frac{1-\gamma_{i_{j+1}}}{\ln 2}-3 A_{i_{j}} \frac{f_{\text {inf }}^{j} r_{j}}{g_{\text {sup }}^{j}} \log _{2}\left(i_{j}+1\right) \\
& -\frac{2 f_{\text {sup }}^{j} r_{j}}{g_{\text {inf }}^{j}} \frac{\log _{2}\left(i_{j}+1\right)-1}{i_{j}+1}-6 A_{i_{j}} \frac{f_{\text {sup }}^{j} r_{j}}{g_{\text {inf }}^{j}}\left(1+\log _{2}\left(\frac{f_{\text {sup }}^{j} r_{j}}{g_{\text {inf }}^{j} d_{j}}\right)\right),
\end{aligned}
$$

где

$$
\gamma_{i_{j}}=\sum_{i=1}^{i_{j}} \frac{1}{i}-\ln i_{j}, \quad A_{i_{j}}=\left(1-a_{j}\right)^{i_{j}}\left(i_{j}+1\right)^{c_{j}}, \quad c_{j}=\left(\left[\frac{1}{a_{j}}\right]+1\right)>1 .
$$


Обозначим через $f_{\text {inf }}, f_{\text {sup }}, g_{\text {inf }}$ и $g_{\text {sup }}$ инфимумы и супремумы функций $f$ и $g$ по всему интервалу $(0,1)$. При любом значении $j$ верны неравенства

$$
\begin{gathered}
\frac{f_{\text {inf }}}{\left[\log _{2} n\right]} \leqslant f_{\text {inf }}\left(b_{j}-a_{j}\right) \leqslant d_{j} \leqslant f_{\text {sup }}\left(b_{j}-a_{j}\right) \leqslant \frac{f_{\text {sup }}}{\left[\log _{2} n\right]}, \\
\frac{g_{\text {inf }}}{\left[\log _{2} n\right]} \leqslant g_{\text {inf }}\left(b_{j}-a_{j}\right) \leqslant r_{j} \leqslant g_{\text {sup }}\left(b_{j}-a_{j}\right) \leqslant \frac{g_{\text {sup }}}{\left[\log _{2} n\right]}, \\
\frac{1}{e} \geqslant a_{j} \geqslant \frac{f_{\text {inf }} g_{\text {inf }}}{f_{\text {sup }} g_{\text {sup }} e} .
\end{gathered}
$$

Значения индекса $i_{j}$ изменяются в пределах от $r_{j} n-n^{2 / 3}$ до $r_{j} n+n^{2 / 3}$. Поэтому при $n$, стремящемся к бесконечности, рассматриваемые значения индекса $i_{j}$ имеют порядок $n / \log _{2} n$. Выражения $A_{i_{j}}$ и $\left(A_{i_{j}} \log _{2}\left(i_{j}+1\right)\right)$ стремятся к нулю при $n$, стремящемся к бесконечности. Выражения $f_{\text {inf }}^{j} / g_{\text {sup }}^{j}$ и $f_{\text {sup }}^{j} / g_{\text {inf }}^{j}$ при любом $j$ ограничены сверху значением $f_{\text {sup }} / g_{\text {inf }}$. Величина $\gamma_{i_{j+1}}$ сходится к константе Эйлера $\gamma$. Отсюда следует, что существует такое натуральное $n_{0}$, что при любом $n$, большем $n_{0}$, выполнено неравенство

$$
\mathbf{M}_{\widetilde{V}}\left(H_{n}^{(f, g)}(\tilde{V}) \mid B_{i_{j}}\right) \geqslant \frac{f_{\text {inf }}^{j} r_{j}}{g_{\text {sup }}^{j}} \log _{2}\left(i_{j}+1\right)-\frac{f_{\text {sup }} r_{j}}{g_{\text {inf }}} \frac{1-\gamma}{\ln 2}-r_{j} .
$$

Подставляя получившиеся оценки в сумму по множеству $J_{j}$, получаем неравенство

$$
\sum_{J_{j}} \mathbf{M}_{\widetilde{V}}\left(H_{n}^{(f, g)}(\tilde{V}) \mid B_{i_{j}}\right) \mathbf{P}\left(B_{i_{j}}\right) \geqslant \sum_{J_{j}}\left(\frac{f_{\text {inf }}^{j}}{g_{\text {sup }}^{j}} r_{j} \log _{2}\left(i_{j}+1\right)-\frac{f_{\text {sup }} r_{j}}{g_{\text {inf }}} \frac{1-\gamma}{\ln 2}-r_{j}\right) \mathbf{P}\left(B_{i_{j}}\right) .
$$

Раскрывая скобки в правой части неравенства и оценивая единицей сумму вероятностей в слагаемых со знаком минус, получаем неравенство

$$
\sum_{J_{j}} \mathbf{M}_{\widetilde{V}}\left(H_{n}^{(f, g)}(\tilde{V}) \mid B_{i_{j}}\right) \mathbf{P}\left(B_{i_{j}}\right) \geqslant \frac{f_{\text {inf }}^{j}}{g_{\text {sup }}^{j}} r_{j} \sum_{J_{j}}\left(\log _{2}\left(i_{j}+1\right) \mathbf{P}\left(B_{i_{j}}\right)\right)-\frac{f_{\text {sup }} r_{j}}{g_{\text {inf }}} \frac{1-\gamma}{\ln 2}-r_{j} .
$$

Если просуммировать по всем индексам $j$ последние два слагаемых в правой части неравенства, то получим оценки

$$
\begin{gathered}
-\sum_{j} r_{j} \geqslant-\int_{0}^{1} g(t) d t \\
-\sum_{j} \frac{f_{\text {sup }} r_{j}}{g_{\text {inf }}} \frac{1-\gamma}{\ln 2} \geqslant-\frac{f_{\text {sup }}}{g_{\text {inf }}} \frac{1-\gamma}{\ln 2} \int_{0}^{1} g(t) d t .
\end{gathered}
$$

Индекс $j$ изменяется в пределах от $r_{j} n-n^{2 / 3}$ до $r_{j} n+n^{2 / 3}$. Поскольку логарифм возрастающая функция, верно неравенство

$$
\begin{aligned}
\frac{f_{\text {inf }}^{j}}{g_{\text {sup }}^{j}} \int_{a_{j}}^{b_{j}} g(t) d t \sum_{J_{j}}\left(\log _{2}\left(i_{j}+1\right)\right) & \mathbf{P}\left(B_{i_{j}}\right) \\
\geqslant & \frac{f_{\text {inf }}^{j}}{g_{\text {sup }}^{j}} \int_{a_{j}}^{b_{j}} g(t) d t \log _{2}\left(\frac{f_{\text {inf }} n}{\left[\log _{2} n\right]}-n^{2 / 3}\right) \sum_{J_{j}} \mathbf{P}\left(B_{i_{j}}\right) .
\end{aligned}
$$


Вероятность того, что запись библиотеки попадет в интервал $\left(a_{j}, b_{j}\right)$ не меньше, чем $g_{\text {inf }} / \log _{2} n$. По лемме 4 получаем, что $\sum_{J_{j}} \mathbf{P}\left(B_{i_{j}}\right)$ стремится к единице.

Покажем, что

$$
\sum_{j=0}^{\log _{2} n} \frac{f_{\text {inf }}^{j}}{g_{\text {sup }}^{j}} \int_{a_{j}}^{b_{j}} g(t) d t \log _{2}\left(\frac{f_{\text {inf }} n}{\log _{2} n}-n^{2 / 3}\right) \gtrsim \log _{2} n .
$$

Двоичный логарифм $\log _{2}\left(f_{\text {inf }} n / \log _{2} n-n^{2 / 3}\right)$ не зависит от индекса $j$, поэтому его можно вынести за скобки. Асимптотически этот логарифм ведет себя как $\log _{2} n$.

Покажем, что

$$
\sum_{j=0}^{\log _{2} n} \frac{f_{\text {inf }}^{j}}{g_{\text {sup }}^{j}} \int_{a_{j}}^{b_{j}} g(t) d t \rightarrow 1
$$

Интеграл $\int_{a_{j}}^{b_{j}} g(t) d t$ можно оценить следующим образом:

$$
g_{\text {inf }}^{j}\left(b_{j}-a_{j}\right) \leqslant \int_{a_{j}}^{b_{j}} g(t) d t \leqslant g_{\text {sup }}^{j}\left(b_{j}-a_{j}\right) .
$$

Подставляя оценку сверху, получаем, что

$$
\sum_{j=0}^{\log _{2} n} \frac{f_{\text {inf }}^{j}}{g_{\text {sup }}^{j}} \int_{a_{j}}^{b_{j}} g(t) d t \leqslant \sum_{j=0}^{\log _{2} n} f_{\text {inf }}^{j}\left(b_{j}-a_{j}\right) \leqslant 1 .
$$

Подставим нижнюю оценку:

$$
\sum_{j=0}^{\log _{2} n} \frac{f_{\text {inf }}^{j}}{g_{\text {sup }}^{j}} \int_{a_{j}}^{b_{j}} g(t) d t \geqslant \sum_{j=0}^{\log _{2} n} \frac{g_{\text {inf }}^{j}}{g_{\text {sup }}^{j}} f_{\text {inf }}^{j}\left(b_{j}-a_{j}\right) .
$$

Выражение $g_{\text {inf }}^{j} / g_{\text {sup }}^{j}$ запишем как

$$
\frac{g_{\text {inf }}^{j}}{g_{\text {sup }}^{j}}=\frac{g_{\text {sup }}^{j}-\left(g_{\text {sup }}^{j}-g_{\text {inf }}^{j}\right)}{g_{\text {inf }}^{j}}=1-\frac{g_{\text {sup }}^{j}-g_{\text {inf }}^{j}}{g_{\text {inf }}^{j}} .
$$

Получаем, что

$$
\sum_{j=0}^{\log _{2} n} \frac{g_{\text {inf }}^{j}}{g_{\text {sup }}^{j}} f_{\text {inf }}^{j}\left(b_{j}-a_{j}\right)=\sum_{j=0}^{\log _{2} n} f_{\text {inf }}^{j}\left(b_{j}-a_{j}\right)-\sum_{j=0}^{\log _{2} n} f_{\text {inf }}^{j}\left(b_{j}-a_{j}\right) \frac{g_{\text {sup }}^{j}-g_{\text {inf }}^{j}}{g_{\text {inf }}^{j}} .
$$

Первое слагаемое в правой части равенства стремится к единице. Покажем, что второе слагаемое стремится к нулю. Верно следующее неравенство:

$$
\sum_{j=0}^{\log _{2} n} f_{\text {inf }}^{j}\left(b_{j}-a_{j}\right) \frac{g_{\text {sup }}^{j}-g_{\text {inf }}^{j}}{g_{\text {inf }}^{j}} \leqslant \frac{f_{\text {sup }}}{g_{\text {inf }}} \sum_{j=0}^{\log _{2} n}\left(b_{j}-a_{j}\right)\left(g_{\text {sup }}^{j}-g_{\text {inf }}^{j}\right) .
$$

Поскольку функция $g$ по условию теоремы интегрируема по Риману, то правая часть неравенства стремится к нулю.

Мы показали, что математическое ожидание нижней энтропийной оценки асимптотически не меньше, чем $\log _{2} n$.

Доказательство леммы закончено. 
По условию теоремы, функции $f$ и $g-$ правильные. Множества $\operatorname{supp}(f)$ и $\operatorname{supp}(g)$ состоят из конечного числа интервалов. Рассмотрим следующие два множества. Первое множество состоит из точек, которые являются концами интервалов из $\operatorname{supp}(f)$, второе множество состоит из точек, которые являются концами интервалов из $\operatorname{supp}(g)$. Точки из объединения этих множеств разбивают интервал $(0,1)$ на подинтервалы таким образом, что на каждом из подинтервалов разбиения функция $f$ либо равна нулю на всем подинтервале, либо на всем подинтервале отлична от нуля. Для функции $g$ ситуация аналогична.

Обозначим через $A_{1}=\left\{\left(u_{1}, v_{1}\right), \ldots,\left(u_{t}, v_{t}\right)\right\}$ множество подинтервалов этого разбиения, на котором функции $f$ и $g$ одновременно отличны от нуля. Записи, которые попали в интервал $\left(u_{j}, v_{j}\right), j=1, \ldots, t$, обозначим через $Y_{1}^{j}, \ldots, Y_{i_{j}}^{j}$. Вероятность попадания запроса в интервал $\left(Y_{(k)}^{j}, Y_{(k+1)}^{j}\right), k=1, \ldots,\left(i_{j}-1\right), j=1, \ldots, t$, обозначим через $P_{k}^{j}$. Для нижней энтропийной оценки $H_{n}^{(f, g)}(\tilde{V})$ верно неравенство

$$
H_{n}^{(f, g)}(\tilde{V}) \geqslant H\left(P_{1}^{1}, \ldots, P_{i_{1}-1}^{1}\right)+\ldots+H\left(P_{1}^{t}, \ldots, P_{i_{t}-1}^{t}\right) .
$$

Обозначим выражение $H\left(P_{1}^{j}, \ldots, P_{i_{j}-1}^{j}\right), j=1, \ldots, t$, через $H_{i_{j}}^{(f, g)}$.

Обозначим через $B_{i_{j}}, 0 \leqslant i_{j} \leqslant n, 1 \leqslant j \leqslant t$, событие, состоящее в том, что в интервале $\left(u_{j}, v_{j}\right)$ ровно $i_{j}$ записей библиотеки. Введем обозначение

$$
r_{j}=\int_{u_{j}}^{v_{j}} g(x) d x
$$

Множество значений индекса $i_{j}$ в пределах от $\left[r_{j} n-n^{2 / 3}\right]$ до $\left[r_{j} n+n^{2 / 3}\right]$ будем обозначать $J_{j}$.

Для математического ожидания нижней энтропийной оценки верно следующее неравенство:

$$
\mathbf{M}_{\widetilde{V}}\left(H_{n}^{(f, g)}(\tilde{V})\right) \geqslant \sum_{J_{1}} \mathbf{M}\left(H_{i_{1}}^{(f, g)} \mid B_{i_{1}}\right) \mathbf{P}\left(B_{i_{1}}\right)+\ldots+\sum_{J_{t}} \mathbf{M}\left(H_{i_{t}}^{(f, g)} \mid B_{i_{t}}\right) \mathbf{P}\left(B_{i_{t}}\right) .
$$

Введем величину

$$
d_{j}=\int_{u_{j}}^{v_{j}} f(t) d t
$$

Обозначим через $\bar{P}_{1}^{j}$ выражение $P_{1}^{j} / d_{j}$. Для функции $H_{i_{j}}^{(f, g)}$ верно следующее неравенство:

$$
H\left(P_{1}^{j}, \ldots, P_{i_{j}-1}^{j}\right) \geqslant d_{j} H\left(\bar{P}_{1}^{j}, \ldots, \bar{P}_{i_{j}-1}^{j}\right) .
$$

Рассмотрим случайную библиотеку $\tilde{V}^{\prime}=\left(Y_{(1)}^{\prime}, Y_{(2)}^{\prime}, \ldots, Y_{\left(i_{j}\right)}^{\prime}\right)$, для которой случайные записи $Y_{i}^{\prime}$ принимают значения из интервала $(0,1)$ и имеют функцию плотности

$$
g^{\prime}(x)=\frac{v_{j}-u_{j}}{r_{j}} g\left(\left(v_{j}-u_{j}\right) x+u_{j}\right)
$$

Рассмотрим запрос $X^{\prime}$, который также принимает значения из интервала $(0,1)$ и имеет функцию плотности

$$
f^{\prime}(x)=\frac{v_{j}-u_{j}}{d_{j}} f\left(\left(v_{j}-u_{j}\right) x+u_{j}\right)
$$


Математическое ожидание нижней энтропийной оценки $H_{i_{j}}^{\left(f^{\prime}, g^{\prime}\right)}\left(\tilde{V}^{\prime}\right)$ имеет ту же асимптотику, что и выражение $H\left(\bar{P}_{1}^{j}, \ldots, \bar{P}_{i_{j}-1}^{j}\right)$. По лемме 6, при $i_{j} \rightarrow \infty$

$$
\mathbf{M}_{\tilde{V}^{\prime}}\left(H_{i_{j}}^{\left(f^{\prime}, g^{\prime}\right)}\left(\tilde{V}^{\prime}\right)\right) \sim \log _{2} i_{j} .
$$

Значение индекса $i_{j}$ изменяется в пределах от $\left[r_{j} n-n^{2 / 3}\right]$ до $\left[r_{j} n+n^{2 / 3}\right]$, то есть при $n \rightarrow \infty$ все рассматриваемые значения $i_{j}$ асимптотически ведут себя как $r_{j} n$. По лемме 4 , сумма $\sum_{J_{j}} \mathbf{P}\left(B_{i_{j}}\right)$ стремится к единице. Учитывая это, получаем, что при $n \rightarrow \infty$

$$
\sum_{J_{j}} \mathbf{M}_{V}\left(H_{j}^{(f, g)}(V) \mid B_{i_{j}}\right) \mathbf{P}\left(B_{i_{j}}\right) \sim d_{j} \log _{2} n .
$$

Просуммировав по всем интервалам множества $A_{1}$, получим, что выражение $H_{n}^{(f, g)}(\tilde{V})$ асимптотически не меньше, чем $\log _{2} n \sum_{j=1}^{t} d_{j}$. Суммирование идет по тем интервалам $A_{1}$, где функции $f$ и $g$ одновременно отличны от нуля. Заметим, что на интервалах, где функция $f$ тождественна равна нулю, а функция $g$ отлична от нуля, значение $d_{j}$ равно нулю. Поэтому

$$
\sum_{j=1}^{t} d_{j}=\int_{\operatorname{supp}(g)} f(t) d t
$$

Получаем, что величина $\mathbf{M}_{\widetilde{V}}\left(T_{n}^{(f, g)}(\tilde{V})\right)$ асимптотически не меньше $\log _{2} n \int_{B} f(t) d t$, где $B=\operatorname{supp}(g)$.

Доказывать, что величина $\mathbf{M}_{\widetilde{V}}\left(T_{n}^{(f, g)}(\tilde{V})\right)$ асимптотически не больше $\log _{2} n \int_{B} f(t) d t$, мы будем с помощью построения ИГ с соответствующей сложностью.

Обозначим

$$
A_{3}=\left\{\left(u_{1}, v_{1}\right), \ldots,\left(u_{t}, v_{t}\right),\left(u_{t+1}, v_{t+1}\right) \ldots,\left(u_{t+m}, v_{t+m}\right)\right\}, \quad m \geqslant 0,
$$

множество интервалов, у которых вероятность попадания в них хотя бы одной записи библиотеки ненулевая. Заметим, что $A_{1} \subset A_{3}$. Обозначим $B_{i_{1}, \ldots, i_{j}}, 0 \leqslant i_{j} \leqslant n$, $j=0,1, \ldots, t+m, i_{1}+\ldots+i_{t+m}=n$, событие, состоящее в том, что интервале $\left(u_{j}, v_{j}\right), i=1, \ldots, t+m$, содержится $i_{j}$ записей библиотеки.

Для этого события построим ИГ $U_{i_{1}, \ldots, i_{j}}$ следующим образом. Сначала с помощью переключателей вида $\breve{p}_{u_{j}}(x), \breve{p}_{v_{j}}(x), j=1, \ldots, t+m$, построим верхнюю часть ИГ, которая представляет собой дерево бинарного поиска глубиной $\log _{2}(2(t+m+1))$. Это дерево определяет, в какой интервал множества $A_{3}$ попал запрос, если запрос не попал в интервал множества $A_{3}$, то в какой промежуток между интервалами попал запрос. К вершинам на последнем уровне этого дерева подсоединим дерево бинарного поиска среди тех записей библиотек, которые попали в проводимость соответствующей вершины.

Во всех промежутках между интервалами множества $A_{3}$ функция $g$ тождественно равна нулю, поэтому вероятность наличия в этом промежутке записи библиотеки равна нулю. Учитывая также, что функция плотности распределения запросов тождественно равна нулю на интервалах множества $A_{3} / A_{1}$, получаем, что математическое ожидание сложности ИГ при условии события $B_{i_{1}, \ldots, i_{j}}$ можно оценить следующим образом:

$$
\left.\mathbf{M}\left(T\left(U_{i_{1}, \ldots, i_{j}}\right) \mid B_{i_{1}, \ldots, i_{j}}\right) \leqslant \log _{2} 2(t+m+1)+d_{j} \sum_{j=1}^{t}\right] \log _{2}\left(i_{j}+1\right)[.
$$


Суммируя по всем события $B_{i_{1}, \ldots, i_{j}}$ получаем, что

$$
\begin{gathered}
\mathbf{M}_{\tilde{V}}\left(T_{n}^{(f, g)}(\tilde{V})\right) \leqslant \log _{2} 2(t+m+1)+d_{1} \sum_{i_{1}=0}^{n} \log _{2}\left(i_{1}+1\right) \mathbf{P}\left(B_{i_{1}}\right)+\ldots \\
+d_{t} \sum_{i_{t}=0}^{n} \log _{2}\left(i_{t}+1\right) \mathbf{P}\left(B_{i_{t}}\right) .
\end{gathered}
$$

Суммы в правой части неравенства оценим следующим образом:

$$
\left.\sum_{i_{j}=0}^{n}\right] \log _{2}\left(i_{j}+1\right)\left[\mathbf{P}\left(B_{i_{j}}\right) \leqslant \log _{2}(n+1) \sum_{i_{j}=0}^{n} \mathbf{P}\left(B_{i_{j}}\right)=\log _{2}(n+1) .\right.
$$

Поэтому при $n \rightarrow \infty$

$$
\mathbf{M}_{\widetilde{V}}\left(T_{n}^{(f, g)}(\tilde{V})\right) \lesssim \log _{2} n \int_{B} f(t) d t,
$$

где $B=\operatorname{supp}(g)$. Доказательство теоремы закончено.

\section{5. Доказательство теоремы 4}

Функцию плотности распределения запросов $f^{\prime}$ и функцию плотности распределения элементов библиотеки $g^{\prime}$ определим следующим образом:

$$
\begin{aligned}
& f^{\prime}(x)= \begin{cases}2, & x \in(0,1 / 2), \\
0 & \text { в противном случае, }\end{cases} \\
& g^{\prime}(x)= \begin{cases}0, & x \in(0,1 / 2), \\
2 & \text { в противном случае. }\end{cases}
\end{aligned}
$$

При любом $n \in \mathbf{N}$ с вероятностью, равной единице, в интервале $(0,1 / 2)$ нет записей библиотеки. Из того, что в интервале $(0,1 / 2)$ нет записей библиотеки, следует, что $\mathbf{P}\left(x \in\left(0, y_{(1)}\right)\right)=1$. Для таких задач поиска идентичных объектов ИГ, реализующий алгоритм последовательного перебора, начиная с записи $y_{(1)}$, имеет сложность 1 . Первое утверждение теоремы доказано.

Возьмем произвольное вещественное число $b>1$. Выберем такое натуральное $m$, что $\log _{2}(1+m)>b$. Рассмотрим функцию энтропии от $m+1$ переменного $H\left(t_{0}, t_{1}, \ldots, t_{m}\right)$; она непрерывна на компакте $\left\{\left(t_{0}, t_{1}, \ldots, t_{m}\right): \sum_{i=0}^{m} t_{i}=1, t_{j} \geqslant 0, j=0, \ldots, m\right\}$ и принимает значения от нуля до $\log _{2}(1+m)$. Поскольку $b \in\left[0, \log _{2}(1+m)\right]$, существует такой набор $\left(t_{0}^{\prime}, t_{1}^{\prime}, \ldots, t_{m}^{\prime}\right)$, что

$$
H\left(t_{0}^{\prime}, t_{1}^{\prime}, \ldots, t_{m}^{\prime}\right)=b, \quad \sum_{i=0}^{m} t_{i}^{\prime}=1 .
$$

Построим искомые функции плотности $f^{\prime \prime}$ и $g^{\prime \prime}$, используя выбранные число $m$ и набор $\left(t_{0}^{\prime}, t_{1}^{\prime}, \ldots, t_{m}^{\prime}\right)$ :

$$
\begin{aligned}
& f^{\prime \prime}(t)=\sum_{i=0}^{m} t_{i}^{\prime} I_{(2 i) /(2 m+1),(2 i+1) /(2 m+1)}(t), \\
& g^{\prime \prime}(t)=\sum_{i=1}^{m} \frac{2 m+1}{m} I_{(2 i-1) /(2 m+1),(2 i) /(2 m+1)}(t),
\end{aligned}
$$


где $I_{Z}(t)$ - характеристическая функция подмножества $Z \subset(0,1)$.

При выбранных функциях плотности вероятность события, когда хотя бы в одном из интервалов $((2 i) /(2 m+1),(2 i+1) /(2 m+1)), i=0,1, \ldots, m$, есть хотя бы одна запись библиотеки, равна нулю. Далее не будем рассматривать это событие.

При $n>m$ для любой случайной библиотеки $\widetilde{V}$ выполнено следующее свойство. Если обозначить через $p_{i}$ вероятность попадания запроса в интервал $\left(y_{(i)}, y_{(i+1)}\right), i=1, \ldots, n$, то ненулевая вероятность соответствует не более, чем $m$ интервалам. Таким образом, с вероятностью, равной единице, величина $T_{n}^{\left(f^{\prime \prime}, g^{\prime \prime}\right)}(\tilde{V})$ не больше, чем $\log _{2}(m+1)$.

При $n>m$ рассмотрим два события. Первое событие состоящее в том, что в каждом из $m$ интервалов $((2 i-1) /(2 m+1),(2 i) /(2 m+1)), i=1, \ldots, m$, есть хотя бы одна запись библиотеки, обозначим через $A_{n}$. Второе событие $B_{n}$ является дополнением $A_{n}$.

Величину $T_{n}^{\left(f^{\prime \prime}, g^{\prime \prime}\right)}(\tilde{V})$ можно оценить с помощью функции энтропии следующим образом:

$$
H\left(p_{0}, p_{1}, \ldots, p_{n}\right) \leqslant T_{n}^{\left(f^{\prime \prime}, g^{\prime \prime}\right)}(V)<H\left(p_{0}, p_{1}, \ldots, p_{n}\right)+2 .
$$

При выполнении события $A_{n}$ вероятности $\left(p_{0}, p_{1}, \ldots, p_{n}\right)$ устроены следующим образом. Только $m$ вероятностей отличны от нуля. Если из отличных от нуля вероятностей составить набор в порядке увеличения значения, то получится перестановка набора $\left(t_{0}^{\prime}, t_{1}^{\prime}, \ldots, t_{m}^{\prime}\right)$. Поскольку функция энтропии симметрична относительно своих переменных, можно записать следующую цепочку тождеств:

$$
H\left(p_{0}, p_{1}, \ldots, p_{n}\right)=H\left(t_{0}^{\prime}, t_{1}^{\prime}, \ldots, t_{m}^{\prime}\right)=b .
$$

При выполнении события $A_{n}$ справедливо неравенство

$$
b \leqslant T\left((0,1), V, \rho=, f^{\prime \prime}\right)<b+2 \text {. }
$$

Покажем, что при увеличении $n$ вероятность события $B_{n}$ стремится к нулю. Обозначим через $B_{n}^{j}, j=1, \ldots, m$, событие, состоящее а том, что в интервале $((2 j-1) /(2 m+1),(2 j) /(2 m+1))$ нет записей библиотеки. Для любого $j=1, \ldots, m$, мера множества $B_{n}^{j}$ равна $(1-1 /(2 m+1))^{n}$. Меру множества $B_{n}$ можно оценить следующим образом:

$$
\mathbf{P}\left(B_{n}\right)<\sum_{j=1}^{m} \mathbf{P}\left(B_{n}^{j}\right)=m\left(1-\frac{1}{2 m+1}\right)^{n} .
$$

Поскольку $m-$ константа, величина $\mathbf{P}\left(B_{n}\right)$ стремится к нулю при увеличении $n$. При $n>m$ справедливо равенство

$$
\mathbf{M}_{\tilde{V}}\left(T_{n}^{\left(f^{\prime \prime}, g^{\prime \prime}\right)}(\tilde{V})\right)=\mathbf{M}_{V}\left(\left(T_{n}^{\left(f^{\prime \prime}, g^{\prime \prime}\right)}(\tilde{V})\right) \mid A_{n}\right) \mathbf{P}\left(A_{n}\right)+\mathbf{M}_{\tilde{V}}\left(\left(T_{n}^{\left(f^{\prime \prime}, g^{\prime \prime}\right)}(\tilde{V})\right) \mid B_{n}\right) \mathbf{P}\left(B_{n}\right) .
$$

При $n>m$ верны неравенства

$$
b \mathbf{P}\left(A_{n}\right) \leqslant \mathbf{M}_{\widetilde{V}}\left(T_{n}^{\left(f^{\prime \prime}, g^{\prime \prime}\right)}(V)\right) \leqslant(b+2) \mathbf{P}\left(A_{n}\right)+\log _{2}(m+1) \mathbf{P}\left(B_{n}\right) .
$$

Поскольку $\mathbf{P}\left(B_{n}\right) \rightarrow 0$ при $n \rightarrow \infty$, получаем, что $\mathbf{P}\left(A_{n}\right) \rightarrow 1$ при $n \rightarrow \infty$. Поэтому существует такое натуральное $n_{0}$, что при любом $n$, большем $n_{0}$,

$$
b \leqslant \mathbf{M}_{\widetilde{V}}\left(T_{n}^{\left(f^{\prime \prime}, g^{\prime \prime}\right)}(\widetilde{V})\right) \leqslant b+2 .
$$

Доказательство теоремы закончено. 


\section{6. Доказательство теоремы 5}

Построим искомые функции плотности $f$ и $g$. Рассмотрим ряд $\sum_{i=2}^{\infty} 1 /\left(i \log _{2}^{2} i\right)$. Этот ряд сходится, поскольку

$$
\begin{aligned}
\sum_{i=2}^{\infty} \frac{1}{i \log _{2}^{2} i} & <\frac{1}{2}+\int_{2}^{\infty} \frac{1}{x \log _{2}^{2} x} d x \\
\int_{2}^{\infty} \frac{1}{x \log _{2}^{2} x} d x & =\ln 2 \int_{2}^{\infty} \frac{1}{\log _{2}^{2} x} d\left(\log _{2} x\right) \\
& =-\ln 2 \int_{2}^{\infty} d\left(\log _{2}^{-1} x\right)=\ln 2 .
\end{aligned}
$$

Обозначим сумму ряда $\sum_{i=2}^{\infty} 1 /\left(i \log _{2}^{2} i\right)$ через $a$. Положим

$$
a_{i}=\frac{1}{2 a(i+2) \log _{2}^{2}(i+2)}, \quad i=0,1, \ldots
$$

Заметим, что

$$
\sum_{i=0}^{\infty} a_{i}=\frac{1}{2}
$$

Используем введенные величины для построения разбиения интервала $(0,1)$ :

$$
\begin{aligned}
\Delta_{0} & =\left(0, a_{0}\right), & \Delta_{1} & =\left(a_{0}, a_{0}+a_{0}\right), \ldots, \\
\Delta_{2 k-1} & =\left(2 \sum_{i=0}^{k-2} a_{i}+a_{k-1}, 2 \sum_{i=0}^{k-1} a_{i}\right), & \Delta_{2 k} & =\left(2 \sum_{i=0}^{k-1} a_{i}, 2 \sum_{i=0}^{k-1} a_{i}+a_{k}\right), \ldots
\end{aligned}
$$

Функцию плотности $f$ определим так, что на нечетных интервалах она равна нулю, а на четных представляет собой равнобедренный треугольник высотой 4. Площадь равнобедренного треугольника на интервале $\Delta_{2 k}, k=0,1, \ldots$, обозначим через $\hat{p}_{k}$ и заметим, что $\hat{p}_{k}=2 a_{k}$. Интеграл функции $f$ по интервалу $(0,1)$ равен единице.

Функция плотности $g$ равна нулю на четных интервалах. На нечетных интервалах $\Delta_{2 k+1}, k=0,1, \ldots$, она имеет вид равнобедренного треугольника высотой

$$
h_{k}=\frac{4 a \log _{2}^{2}(k+2)}{k+1} .
$$

Площадь этого треугольника обозначим через $q_{k}$, очевидно,

$$
q_{k}=\frac{1}{(k+1)(k+2)} \text {. }
$$

Ряд $\sum_{i=0}^{\infty} q_{i}$ сходится, и сумма его равна единице.

Покажем, что верна верхняя оценка в утверждении теоремы. Рассмотрим событие, состоящее в том, что ни в одном из интервалов с номером $2 k+1$, где $k>n^{2}-2$, нет записей библиотеки и обозначим его через $A_{n}$. Вероятность того, что запись библиотеки не попадет в эти интервалы, равна

$$
\sum_{k=0}^{n^{2}-2} \frac{1}{(k+1)(k+2)}=1-\frac{1}{2}+\frac{1}{2}-\frac{1}{3} \ldots-\frac{1}{n^{2}}=1-\frac{1}{n^{2}} .
$$


Вероятность события $A_{n}$ равна

$$
\mathbf{P}\left(A_{n}\right)=\left(1-\frac{1}{n^{2}}\right)^{n}=\left(\left(1-\frac{1}{n^{2}}\right)^{n^{2}}\right)^{1 / n}
$$

и при $n \rightarrow \infty$ стремится к единице.

Математическое ожидание сложности рассматриваемого класса задач можно оценить с помощью энтропии

$$
\mathbf{M}_{\widetilde{V}}\left(T_{n}^{(f, g)}(\tilde{V})\right)<\mathbf{M}_{\widetilde{V}}\left(H_{n}^{(f, g)}(\tilde{V})\right)+2 .
$$

Используя условные математические ожидания, математическое ожидание энтропии можно записать следующим образом:

$$
\mathbf{M}_{\widetilde{V}}\left(H_{n}^{(f, g)}(\tilde{V})\right)=\mathbf{M}_{\widetilde{V}}\left(H_{n}^{(f, g)}(\tilde{V}) \mid A_{n}\right) \mathbf{P}\left(A_{n}\right)+\mathbf{M}_{\widetilde{V}}\left(H_{n}^{(f, g)}(\tilde{V}) \mid \bar{A}_{n}\right) \mathbf{P}\left(\bar{A}_{n}\right) .
$$

Для функции энтропии справедливо неравенство [5]

$$
H\left(t_{1}, \ldots, t_{n-k}, t_{n-k+1}, \ldots, t_{n}\right) \leqslant H\left(t_{1}, \ldots, t_{n-k}, q\right),
$$

где $q=t_{n-k+1}+\ldots+t_{n}$. Поэтому при выполнения события $A_{n}$

$$
H_{n}^{(f, g)}(\tilde{V}) \leqslant H\left(\hat{p}_{0}, \hat{p}_{1}, \ldots, \hat{p}_{n^{2}+1}, 1-\sum_{i=0}^{n^{2}+1} \hat{p}_{i}\right)<-\sum_{i=0}^{n^{2}+1} \hat{p}_{i} \log _{2} \hat{p}_{i}+1 .
$$

Подставляя

$$
\hat{p}_{i}=\frac{1}{a(i+2) \log _{2}^{2}(i+2)}, \quad i=0,1, \ldots,
$$

и раскрывая скобки, получим, что

$$
-\sum_{i=0}^{n^{2}+1} \hat{p}_{i} \log _{2} \hat{p}_{i}<\frac{1}{a} \sum_{i=2}^{n^{2}+3} \frac{1}{i \log _{2} i}+\frac{1}{a} \sum_{i=2}^{n^{2}+3} \frac{\log _{2} \log _{2}^{2} i}{i \log _{2}^{2} i}+\left|\log _{2} a\right| .
$$

Суммы можно оценить следующим способом:

$$
\begin{gathered}
\sum_{i=2}^{n^{2}+3} \frac{1}{i \log _{2} i}<1+\int_{2}^{n^{2}+3} \frac{1}{x \log _{2} x} d x, \\
\sum_{i=2}^{n^{2}+3} \frac{\log _{2} \log _{2}^{2} i}{i \log _{2}^{2} i}<1+\int_{2}^{n_{2}+3} \frac{2 \log _{2} \log _{2} x}{x \log _{2}^{2} x} d x .
\end{gathered}
$$

Преобразуем первый интеграл:

$$
\int_{2}^{n^{2}+3} \frac{1}{x \log _{2} x} d x=\ln 2 \int_{2}^{n^{2}+3} \frac{1}{x \ln x} d x=\ln 2 \int_{2}^{n^{2}+3} d(\ln \ln x) .
$$

Этот интеграл имеет асимптотику $\ln 2 \ln \ln n$. Значение второго интеграла ограничено некоторой константой, поскольку

$$
\int_{2}^{\infty} \frac{2 \log _{2} \log _{2} x}{x \log _{2}^{2} x} d x=\ln 2 \int_{2}^{\infty} \frac{2 \log _{2} \log _{2} x}{\log _{2}^{2} x} d \log _{2} x=\ln 2 \int_{1}^{\infty} \frac{2 \log _{2} y}{y^{2}} d y<\infty .
$$


Учитывая все полученные оценки, находим, что

$$
\mathbf{M}_{V}\left(T_{n}^{(f, g)}(V)\right) \lesssim \frac{1}{a} \ln 2 \ln \ln n .
$$

Покажем, что верна нижняя асимптотическая оценка в утверждении теоремы. Рассмотрим событие, состоящее в том, что в каждом из интервалов $\Delta_{2 k+1}, k=0,1, \ldots,\left[\log _{2} n\right]$, есть хотя бы одна запись библиотеки, и обозначим его через $B_{n}$. Вероятность этого события можно оценить следующим образом. Рассмотрим событие, состоящее в том, что пуст хотя бы один из отрезков $\Delta_{2 k+1}, k=0,1, \ldots,\left[\log _{2} n\right]$, и обозначим его через $C_{n}$. Тогда

$$
\mathbf{P}\left(B_{n}\right)=1-\mathbf{P}\left(C_{n}\right) .
$$

Справедливо неравенство

$$
\mathbf{P}\left(C_{n}\right)<\sum_{s=1}^{\left[\log _{2} n\right]} \mathbf{P}(\{\text { пуст отрезок с номером } s\}) .
$$

Поскольку

$$
\mathbf{P}(\{\text { пуст отрезок с номером } s\})=\left(1-q_{s}\right)^{n},
$$

где

верна оценка

$$
q_{s}=\frac{1}{(s+1)(s+2)}, \quad s=1, \ldots,\left[\log _{2} n\right], \quad q_{s}>\frac{1}{\left(\log _{2} n+2\right)^{2}},
$$

$$
\mathbf{P}\left(B_{n}\right)>1-\log _{2} n\left(1-\frac{1}{\left(\log _{2} n+2\right)^{2}}\right)^{n} .
$$

Таким образом, $\mathbf{P}\left(B_{n}\right) \rightarrow 1$ при $n \rightarrow \infty$.

Математическое ожидание сложности рассматриваемого класса задач можно оценить с помощью энтропии:

$$
\mathbf{M}_{\widetilde{V}}\left(T_{n}^{(f, g)}(\tilde{V})\right) \geqslant \mathbf{M}_{\widetilde{V}}\left(H_{n}^{(f, g)}(\tilde{V})\right) .
$$

Используя условные математические ожидания, математическое ожидание энтропии можно оценить следующим образом:

$$
\mathbf{M}_{\widetilde{V}}\left(H_{n}^{(f, g)}(\tilde{V})\right)>\mathbf{M}_{\widetilde{V}}\left(H_{n}^{(f, g)}(\tilde{V}) \mid B_{n}\right) \mathbf{P}\left(B_{n}\right) .
$$

Нижняя энтропийная оценка при выполнении события $B_{n}$ всегда содержит слагаемое

$$
-\sum_{i=0}^{\left[\log _{2} n\right]} \hat{p}_{i} \log _{2} \hat{p}_{i}>-\left|\log _{2} a\right|+\frac{1}{a} \sum_{i=2}^{\left[\log _{2} n\right]} \frac{1}{i \log _{2} i} .
$$

Сумма $\sum_{i=2}^{\left[\log _{2} n\right]} 1 /\left(i \log _{2} i\right)$ имеет такую же асимптотику, что и интеграл

$$
\int_{2}^{\left[\log _{2} n\right]} \frac{1}{x \log _{2} x} d x=\ln 2 \int_{2}^{\left[\log _{2} n\right]} d(\ln \ln x) \sim \ln 2 \ln \ln \ln n .
$$

Учитывая все полученные оценки, приходим к оценке

$$
\mathbf{M}_{\widetilde{V}}\left(T_{n}^{(f, g)}(\tilde{V})\right) \gtrsim \frac{1}{a} \ln 2 \ln \ln \ln n .
$$

Доказательство теоремы закончено. 


\section{Список литературы}

1. Гасанов Э. Э., Кудрявцев В. Б., Теория хранения и поиска информации. Физматлит, Москва, 2002.

2. Кучеренко Н. С., Сложность поиска идентичных объектов в случайных базах данных. Интеллектуальные системы (2007) 11, №1-4, 495-516.

3. Кучеренко Н. С., О промежуточных функциях роста сложности поиска для случайных баз данных. Интеллектуальные системы (2009) 13, №1-4, 361-395.

4. Феллер В., Введение в теорию вероятностей и ее приложения, 1-2. Мир, Москва, 1967.

5. Кнут Д. Э., Искусство программирования, 3. Вильямс, Москва, 2000.

6. Knuth D. E., Optimum binary search trees. Acta Informatica (1971) 1, 14-25.

7. Gilbert E. N., Moore E. F., Variable-length binary encodings. Bell System Tech. J. (1959) 38, 933-968.

8. Garsia A. M., Wachs M. L., A new algorithm for minimum cost binary trees. SIAM J. Comput. (1977) 6, 622-642. 